\title{
Note on the space group selection rule for closed strings on orbifolds
}

\author{
Saúl Ramos-Sánchez ${ }^{a, 1}$ and Patrick K.S. Vaudrevange ${ }^{b}$ \\ ${ }^{a}$ Instituto de Física, Universidad Nacional Autónoma de México, \\ POB 20-364, Cd. Mx. 01000, México \\ ${ }^{b}$ Physik Department T75, Technische Universität München, \\ James-Franck-Straße, 85748 Garching, Germany \\ E-mail: ramos@fisica.unam.mx, patrick.vaudrevange@tum.de
}

ABSTRACT: It is well-known that the space group selection rule constrains the interactions of closed strings on orbifolds. For some examples, this rule has been described by an effective Abelian symmetry that combines with a permutation symmetry to a non-Abelian flavor symmetry like $D_{4}$ or $\Delta(54)$. However, the general case of the effective Abelian symmetries was not yet fully understood. In this work, we formalize the computation of the Abelian symmetry that results from the space group selection rule by imposing two conditions only: (i) well-defined discrete charges and (ii) their conservation. The resulting symmetry, which we call the space group flavor symmetry $D_{S}$, is uniquely specified by the Abelianization of the space group. For all Abelian orbifolds with $\mathcal{N}=1$ supersymmetry we compute $D_{S}$ and identify new cases, for example, where $D_{S}$ contains a $\mathbb{Z}_{2}$ dark matterparity with charges 0 and 1 for massless and massive strings, respectively.

KeYwords: Discrete Symmetries, Superstrings and Heterotic Strings

ARXIV EPRINT: 1811.00580

\footnotetext{
${ }^{1}$ Corresponding author.
} 


\section{Contents}

1 Introduction 1

2 Space groups and orbifolds $\quad 2$

2.1 The space group 3

2.2 Presentation of the space group 4

2.3 Geometrical orbifolds and closed strings 5

2.4 The space group selection rule 6

3 Consequences of the space group selection rule $\quad 7$

$\begin{array}{ll}3.1 \text { Conditions on the effective symmetry } & 7\end{array}$

3.2 Consequences of the conditions 8

3.3 Space group selection rule and the Abelianization of the space group 10

3.4 Direct computation 10

3.5 Space group selection rule and remnant discrete symmetries from spontaneous symmetry breaking 12

$\begin{array}{lll}3.6 & \text { Examples } & 13\end{array}$

$\begin{array}{lll}3.6 .1 & \mathbb{Z}_{3} \text { space group in } D=2 \text { dimensions } & 13\end{array}$

3.6.2 Space group with freely-acting shift: $\mathbb{Z}_{2} \times \mathbb{Z}_{2}-5-1 \quad 14$

3.6.3 Space group with roto-translation: $\mathbb{Z}_{2} \times \mathbb{Z}_{2}-2-5 \quad 15$

4 Results $\quad 17$

4.1 Discrete anomalies of the SG flavor symmetry $D_{S} \quad 25$

5 Conclusions $\quad 25$

\section{Introduction}

One of the main goals of string phenomenology is to build a realistic string model that describes and explains the origin of the particles of the Standard Model (SM) and their interactions, as well as of the standard cosmology encoded in the $\Lambda \mathrm{CDM}$ model. In this sense, the compactification of the heterotic string on six-dimensional orbifolds $[1,2]$ can be seen as a promising string scenario. It has led to very large sets of semi-realistic string models, which, among other features, reproduce the particle content of the SM $[3,4]$ or of its supersymmetric extensions [5-12].

Having large sets of semi-realistic orbifold models, the next step is to improve the phenomenological constraints on these models. This can be achieved by a detailed study of the low-energy effective field theory in these constructions. First of all, this requires the identification and understanding of all symmetries that govern the interactions of closed strings 
on orbifolds. These symmetries include gauge symmetries, discrete $R$-symmetries [13-15], target-space modular symmetries [16-19] and Abelian discrete symmetries that arise from the so-called space group selection rule [20, 21]. The latter ones are the main focus of this work: the space group selection rule sets the geometric conditions under which closed strings can split and join while propagating on the surface of an orbifold. These geometric conditions depend on the geometrical orbifold under consideration, which is specified by the space group $S$. Then, for a given space group $S$ one can rephrase the space group selection rule as an effective discrete symmetry which we denote by $D_{S}$ in the following.

The phenomenological relevance of $D_{S}$ has been emphasized by showing that they are essential ingredients of the non-Abelian flavor symmetries realized in the effective field theory of orbifold compactifications [12, 22, 23]. Therefore, we call the Abelian symmetry $D_{S}$ the space group (SG) flavor symmetry. Unfortunately, the nature of the SG flavor symmetry $D_{S}$ has not been fully understood. Furthermore, the computation of $D_{S}$ has only been restricted to the interactions of massless strings, even though massive strings may also play a crucial role for the phenomenology in orbifold compactifications, for example for CP violation due to the presence of heavy string modes [24].

In this work, we aim at completing the study of the SG flavor symmetries arising from the space group selection rule for closed strings on orbifolds. After an introduction to space groups, orbifolds and the space group selection rule in section 2, we formalize in section 3 the computation of the SG flavor symmetry $D_{S}$ by imposing two physical conditions only: $D_{S}$ must be as symmetry with well-defined discrete charges for closed string states and discrete charges must be conserved. This fixes the SG flavor symmetry uniquely via the so-called Abelianization of the space group $S$. After exemplifying the computation, we present in section 4 the SG flavor symmetries $D_{S}$ for all space groups $S$ of six-dimensional orbifold geometries with Abelian point groups and $\mathcal{N}=1$ supersymmetry as classified in refs. $[25,26]$. As a consistency check, we verify that all discrete anomalies of the SG flavor symmetry $D_{S}$ are universal and, hence, can be canceled by a discrete version of the universal Green-Schwarz mechanism [27].

Interestingly, we identify new SG flavor symmetries that act differently on massless and massive strings. In other words, the full string spectrum is subject to a larger symmetry group compared to the massless spectrum. In order to highlight possible phenomenological consequences of this, we present in section 3.6.2 a detailed example for a specific $\mathbb{Z}_{2} \times \mathbb{Z}_{2}$ orbifold. There, the $S G$ flavor symmetry contains a $\mathbb{Z}_{4}$ factor that acts as a $\mathbb{Z}_{2}$ symmetry if restricted to massless strings only. As we show, such a symmetry can be used to define a $\mathbb{Z}_{2}$ dark matter parity, where certain massive strings can only be produced and annihilated in pairs.

\section{Space groups and orbifolds}

In this section we give a detailed review on space groups and their resulting orbifold geometries. We highlight an unconventional approach to use a so-called presentation of a space group, i.e. a way to define a space group by abstract generators and a set of relations among them without explicitly writing out neither a basis of lattice vectors nor the rotation 
matrices. In addition, we briefly discuss closed strings on orbifolds with a focus on those properties that are relevant for the space group selection rule of interacting strings.

\subsection{The space group}

A general element $g$ of a $D$-dimensional space group $S$ can be written as

$$
g=(\rho, \lambda) \in S
$$

We will mostly consider the case $D=6$ in order to compactify the heterotic string from ten to four dimensions. By definition, the space group element $g$ acts on the $D$ extradimensional coordinates $y \in \mathbb{R}^{D}$ as

$$
y \stackrel{g}{\mapsto}(\rho, \lambda) y=\rho y+\lambda,
$$

where the so-called twist $\rho \in \mathrm{O}(D)$ is a $D \times D$ rotation matrix (if $\operatorname{det}(\rho)=1$ ) or reflection matrix (if $\operatorname{det}(\rho)=-1$ ) and the vector $\lambda \in \mathbb{R}^{D}$ yields a translation. Consequently, two space group elements $g_{1}=\left(\rho_{1}, \lambda_{1}\right)$ and $g_{2}=\left(\rho_{2}, \lambda_{2}\right)$ multiply as

$$
\left(\rho_{1}, \lambda_{1}\right)\left(\rho_{2}, \lambda_{2}\right)=\left(\rho_{1} \rho_{2}, \rho_{1} \lambda_{2}+\lambda_{1}\right) .
$$

It follows that $(\mathbb{1}, 0)=\mathbb{1}_{S}$ is the identity element of $S$ and the inverse element of $(\rho, \lambda)$ is given by

$$
(\rho, \lambda)^{-1}=\left(\rho^{-1},-\rho^{-1} \lambda\right) .
$$

Now we can define a $D$-dimensional space group $S$ by specifying finitely many generators: first, one chooses a $D$-dimensional torus lattice $\Lambda_{D}$, which is generated by $D$ linear independent translations $T_{i}$, i.e.

$$
T_{i}=\left(\mathbb{1}, e_{i}\right) \quad \text { for } \quad i=1, \ldots, D,
$$

where the basis vectors $e_{i}$ are given by the columns of a vielbein $e$. In addition, there are generators of the form

$$
(\rho, \lambda) \in S \text { with } \rho \neq \mathbb{1} .
$$

As a remark, if $\lambda \notin \Lambda_{D}$ in eq. (2.6) such generators are referred to as roto-translations. On the other hand, if $\lambda \in \Lambda_{D}$ one can choose a pure rotation as an alternative generator to eq. (2.6), i.e.

$$
(\rho, 0) \in S .
$$

As we will be dealing with space groups with at most two rotational generators, we will label them by $g_{\theta}, g_{\omega} \in S$, where

$$
g_{\theta}=\left(\theta, \lambda_{\theta}\right) \text { and } g_{\omega}=\left(\omega, \lambda_{\omega}\right)
$$

The space group $S$ must close under multiplication. For example,

$$
(\rho, \lambda)\left(\mathbb{1}, e_{i}\right)(\rho, \lambda)^{-1}=\left(\mathbb{1}, \rho e_{i}\right) \stackrel{!}{\in} S
$$


and, consequently, $\rho e_{i}$ must be from the $D$-dimensional lattice $\Lambda_{D}$. In other words, the twist $\rho$ of any space group element has to be an automorphism of the lattice $\Lambda_{D}$. Thus, one can always find a matrix $\hat{\rho}$ from $\operatorname{GL}(D, \mathbb{Z})$ such that

$$
\rho e=e \hat{\rho} .
$$

$\hat{\rho}=e^{-1} \rho e$ is called the twist in the lattice basis. Since $\rho \in \mathrm{O}(D)$, we find the condition

$$
\hat{\rho}^{T} G \hat{\rho}=G,
$$

on the $D$-dimensional torus metric $G=e^{T} e$.

The twists $\rho$ form a finite group $P$, called the point group. Its elements act crystallographically: they map the lattice $\Lambda_{D}$ to itself. As $P$ is finite, each element $\rho \in P$ has to have finite order, i.e. there exists a smallest integer $N_{\rho} \in \mathbb{N}$ such that

$$
\rho^{N_{\rho}}=\mathbb{1}
$$

A point group can be both, Abelian or non-Abelian. If $P$ is Abelian, it is isomorphic to the cyclic group (or to the direct product of several cyclic groups). In the following we restrict ourselves to Abelian point groups which preserve $\mathcal{N}=1$ supersymmetry, being either $\mathbb{Z}_{M}$ or $\mathbb{Z}_{M} \times \mathbb{Z}_{N}$.

\subsection{Presentation of the space group}

The commutator of two space group elements $g, h \in S$ is defined as

$$
[g, h]=g^{-1} h^{-1} g h .
$$

Then, since we restrict ourselves to Abelian point groups, the commutator of any pair of elements $g, h \in S$ is always a pure translation $\lambda_{(g, h)} \in \Lambda_{D}$, i.e.

$$
[g, h]=\left(\mathbb{1}, \lambda_{(g, h)}\right)
$$

Following ref. [29], we can specify a space group $S$ uniquely by a presentation that involves all relations between all generators. For example, in the case of a $\mathbb{Z}_{M} \times \mathbb{Z}_{N}$ point group we have the following generators of $S$ : the rotations (or roto-translations) $g_{\theta}=\left(\theta, \lambda_{\theta}\right)$ and $g_{\omega}=\left(\omega, \lambda_{\omega}\right)$, and the translations $T_{i}=\left(\mathbb{1}, e_{i}\right), i=1, \ldots, D$. Then, all relations are given by

$$
\begin{aligned}
g_{\theta}^{M} & =\left(T_{1}\right)^{a_{(\theta, 1)}} \ldots\left(T_{D}\right)^{a_{(\theta, D)}}, \\
g_{\omega}^{N} & =\left(T_{1}\right)^{a_{(\omega, 1)}} \ldots\left(T_{D}\right)^{a_{(\omega, D)}}, \\
{\left[g_{\theta}, g_{\omega}\right] } & =\left(T_{1}\right)^{a_{1}} \ldots\left(T_{D}\right)^{a_{D}} \\
{\left[T_{i}, T_{j}\right] } & =\mathbb{1}_{S} \\
{\left[g_{\theta}, T_{i}\right] } & =\left(T_{1}\right)^{b_{(i, 1)}} \ldots\left(T_{D}\right)^{b_{(i, D)}}, \\
{\left[g_{\omega}, T_{i}\right] } & =\left(T_{1}\right)^{c_{(i, 1)}} \ldots\left(T_{D}\right)^{c_{(i, D)}},
\end{aligned}
$$


where $M$ and $N$ denote the order of $\theta$ and $\omega$, respectively, and $a_{(\theta, i)}, a_{(\omega, i)}, a_{i}, b_{(i, j)}, c_{(i, j)} \in \mathbb{Z}$ for $i, j=1, \ldots, D$. A few remarks are in order: the right-hand sides of eqs. (2.15a), (2.15b) and $(2.15 \mathrm{c})$ are non-trivial only in the case where $g_{\theta}=\left(\theta, \lambda_{\theta}\right)$ or $g_{\omega}=\left(\omega, \lambda_{\omega}\right)$ are rototranslations, i.e. $\lambda_{\theta} \notin \Lambda_{D}$ or $\lambda_{\omega} \notin \Lambda_{D}$. Furthermore, two translations $T_{i}=\left(\mathbb{1}, e_{i}\right)$ and $T_{j}=\left(\mathbb{1}, e_{j}\right)$ necessarily commute, see eq. (2.15d). Finally, the action of a twist (e.g. $\theta \in P$ ) on the lattice (e.g. $\hat{\theta}=e^{-1} \theta e$, see eq. (2.10)) is uniquely specified by eqs. (2.15e) and (2.15f). In detail, comparing

$$
\left[g_{\theta}, T_{i}\right]=\left(\mathbb{1},\left(\mathbb{1}-\theta^{-1}\right) e_{i}\right),
$$

with eq. (2.15e) we obtain $\left(\hat{\theta}^{-1}\right)_{i j}=\delta_{i j}-b_{(j, i)}$.

Now, in order to write down a presentation of $S$, we rewrite each relation (2.15) such that one has the identity element $\mathbb{1}_{S}$ on the right-hand side. For example, we modify eq. (2.15a) to

$$
g_{\theta}^{M}\left(T_{1}\right)^{-a_{(\theta, 1)}} \ldots\left(T_{D}\right)^{-a_{(\theta, D)}}=\mathbb{1}_{S} .
$$

Then, we suppress the identity element $\mathbb{1}_{S}$ and do the same for all relations (2.15). Consequently, a presentation of the space group $S$ reads

$$
\left.S=\left\langle g_{\theta}, g_{\omega}, T_{1}, \ldots, T_{D}\right| g_{\theta}^{M}\left(T_{1}\right)^{-a_{(\theta, 1)}} \ldots\left(T_{D}\right)^{-a_{(\theta, D)}}, \ldots, \text { all relations }\right\rangle .
$$

One can use the presentation of a space group $S$ to uniquely specify $S$ without writing out the torus vielbein $e$ and the twist matrices explicitly. Indeed, the relations (2.18) contain all information about the space group $S$. This fact is used in section 3 where we discuss the effective symmetries arising from the space group selection rule.

\subsection{Geometrical orbifolds and closed strings}

Having defined the space group $S$, a $D$-dimensional orbifold is defined geometrically as a quotient space $O=\mathbb{R}^{D} / S$ using the equivalence relation

$$
y_{1} \sim y_{2} \quad \Leftrightarrow \quad \exists g \in S \text { such that } y_{1}=g y_{2},
$$

for $y_{1}, y_{2} \in \mathbb{R}^{D}$. In words, two points $y_{1}$ and $y_{2}$ from $\mathbb{R}^{D}$ are identified on the orbifold $O$ if there exists a space group element $g \in S$ that maps $y_{2}$ to $y_{1}$.

A closed string on an orbifold is characterized by the so-called constructing element $g=(\rho, \lambda) \in S$ that specifies the boundary condition for the string to close up to the action of $g$. For example, considering the worldsheet boson $X(\tau, \sigma)$ with worldsheet time and space coordinates $\tau$ and $\sigma \in[0,1]$, respectively, we impose the boundary condition

$$
X(\tau, \sigma+1) \stackrel{!}{=} g X(\tau, \sigma)=\rho X(\tau, \sigma)+\lambda .
$$

If $\rho \neq \mathbb{1}$ the boundary condition eq. (2.20) describes a so-called twisted string that is localized at the fixed point of $g$. On the other hand, if $\rho=\mathbb{1}$ the boundary condition eq. (2.20) describes a so-called untwisted string, which in general can be massless only if the winding vanishes, $\lambda=0$. 
Since $h X(\tau, \sigma)$ and $X(\tau, \sigma)$ are identified on the orbifold for all $h \in S$, see eq. (2.19), the boundary condition eq. (2.20) with constructing element $g \in S$ and the corresponding one with constructing element $h g h^{-1} \in S$ describe the same string on the orbifold. Hence, a closed string with constructing element $g \in S$ is associated to the conjugacy class

$$
[g]=\left\{h g h^{-1} \mid \text { for all } h \in S\right\} .
$$

The resulting closed string state is denoted by $|[g]\rangle$.

Conventionally, one (ambiguously) labels an orbifold by the abstract finite group that is isomorphic to its point group $P$, for example $\mathbb{Z}_{6}$ (and sometimes additional labels to distinguish between different representations of the same abstract finite group, for example $\mathbb{Z}_{6}$-I or $\mathbb{Z}_{6}$-II). In terms of the modern nomenclature (see e.g. $[25,26]$ ), this corresponds to the so-called $\mathbb{Q}$-class. For a given $\mathbb{Q}$-class, there can be several inequivalent torus lattices $\Lambda_{D}$, called $\mathbb{Z}$-classes. Furthermore, for a given $\mathbb{Z}$-class there can be several inequivalent roto-translations, called affine classes. Then, $\mathbb{Z}$ - and affine classes are consecutively enumerated. For example, the space group $\mathbb{Z}_{2} \times \mathbb{Z}_{2}-2-5$ from table 1 belongs to the $\mathbb{Q}$-class $\mathbb{Z}_{2} \times \mathbb{Z}_{2}$, therein to the second $\mathbb{Z}$-class and, finally, therein to the fifth affine class.

\subsection{The space group selection rule}

Let us denote a coupling among $L$ closed string states $\left|\left[g_{a}\right]\right\rangle, a=1, \ldots, L$, moving on an orbifold by

$$
\left|\left[g_{1}\right]\right\rangle\left|\left[g_{2}\right]\right\rangle \cdots\left|\left[g_{L}\right]\right\rangle
$$

where the closed string states are characterized by the conjugacy classes $\left[g_{a}\right]$ of their constructing elements $g_{a} \in S$.

In the underlying CFT of string theory, the amplitude of such couplings of string states is given by the $L$-point correlation functions $[20,21]\left\langle V^{(1)} V^{(2)} \cdots V^{(L)}\right\rangle$ among their corresponding vertex operators $V^{(a)}$, which contain all quantum numbers of the strings, including their boundary conditions given by $g_{a}$. These correlation functions are non-zero only if certain conditions on the quantum numbers encoded in the vertex operators are fulfilled. These constraints are known as string selection rules.

In particular, the so-called space group selection rule indicates under which circumstances the boundary conditions of certain closed strings are compatible, so that they can combine together and still render a closed string. As an example, consider two closed strings with constructing elements $g_{1}$ and $g_{2}$. When these incoming strings merge, they yield an outgoing string with constructing element $g_{3}=g_{1} g_{2}$. In terms of three incoming strings this condition reads

$$
\prod_{a=1}^{L} g_{a}=\mathbb{1}_{S},
$$

for $L=3$. This is schematically represented in figure 1 .

As a closed string is not represented by a single constructing element $g_{a}$ but by the full conjugacy class $\left[g_{a}\right]$, it suffices to choose one element of each of the conjugacy classes 


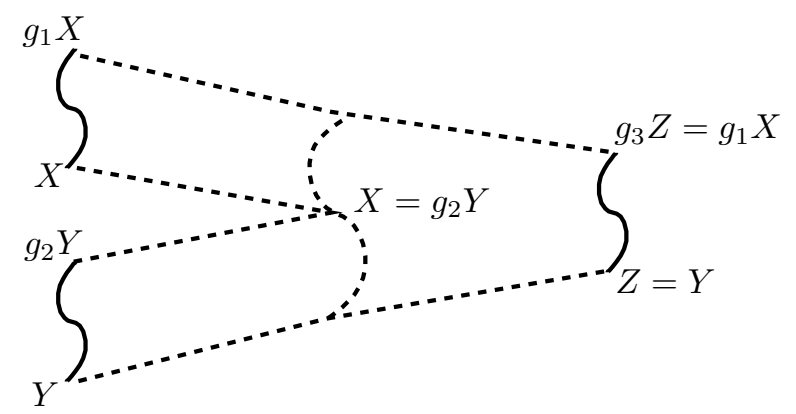

Figure 1. Two incoming strings combine to yield an outgoing string. The equalities are requirements for the coupling to occur. Combining these conditions leads to the space group selection rule on the corresponding constructing elements, $g_{3} Y=g_{1} g_{2} Y$.

of the constructing elements to satisfy the space group selection rule. That is, for $L$-point couplings one can choose elements $h_{a} \in S$, such that

$$
\prod_{a=1}^{L} h_{a} g_{a} h_{a}^{-1}=\mathbb{1}_{S}
$$

in order to obtain a non-vanishing coupling among the states $\left|\left[g_{a}\right]\right\rangle$. As a remark, if eq. (2.24) is satisfied, then the order of string states in eq. (2.22) does not matter.

Depending on the complexity of the space group $S$ under consideration, the space group selection rule is difficult to apply as one has to check eq. (2.24) for all possible choices $h_{a} \in S$ for $a=1, \ldots, L$. Hence, we want to identify the effective SG flavor symmetry $D_{S}$ that incorporates the space group selection rule without this ambiguity. Such a symmetry has to fulfill certain conditions that we now discuss.

\section{Consequences of the space group selection rule}

In this section, we derive the SG flavor symmetry $D_{S}$ that emerges from the geometric restrictions on the interactions of closed strings while moving on the surface of an orbifold.

\subsection{Conditions on the effective symmetry}

We look for a mapping, denoted by $s$, from the space group $S$ to a discrete group $D_{S}$, i.e. $s: S \rightarrow D_{S}$, with the following two properties

(i) $s$ is a class function:

$$
s\left(h g h^{-1}\right)=s(g) \quad \text { for all } h, g \in S \text {. }
$$

(ii) $s$ is a representation of the space group $S$ :

$$
s\left(g_{1}\right) s\left(g_{2}\right)=s\left(g_{1} g_{2}\right),
$$


Let us briefly discuss some immediate implications of these conditions. A necessary condition for the space group selection rule (2.24) to be satisfied is given by

$$
s\left(g_{1}\right) \cdots s\left(g_{L}\right)=s\left(\mathbb{1}_{S}\right),
$$

which follows from eq. (2.24) by applying $s$ on both sides and using our conditions (i) and (ii). Thus, we arrive at a much simpler version of the space group selection rule, where the ambiguity of choosing the elements $h_{a} \in S$ for $a=1, \ldots, L$ has been eliminated. Moreover, from a physical point of view, condition (ii) can be understood as a discrete charge conservation, where $s\left(g_{a}\right)$ corresponds to the discrete charge of a string state $\left|\left[g_{a}\right]\right\rangle$ with constructing element $g_{a}$, i.e.

$$
\left|\left[g_{a}\right]\right\rangle \mapsto s\left(g_{a}\right)\left|\left[g_{a}\right]\right\rangle,
$$

for $a=1, \ldots, L$. Condition (i) ensures that the discrete charge corresponding to $s\left(g_{a}\right)$ is uniquely defined as a string state $\left|\left[g_{a}\right]\right\rangle$ with constructing element $g_{a} \in S$ is characterized by the conjugacy class $\left[g_{a}\right]$, see section 2.3.

Using the conditions (i) and (ii) of eqs. (3.1) and (3.2), it turns out that $s(g)$ (and consequently the SG flavor symmetry $D_{S}$ ) is fully specified for all space groups $S$. In the remainder of this paper we will compute our main result: the space group flavor symmetries $D_{S}$ for all six-dimensional orbifold geometries with Abelian point groups and $\mathcal{N}=1$ supersymmetry, as classified in refs. [25, 26].

As a remark, if a coupling is not invariant under $D_{S}$ then the space group selection rule is also not fulfilled. However, eq. (3.3) is a necessary condition but not sufficient: there are cases, where a coupling is invariant under $D_{S}$, i.e. eq. (3.3) is satisfied, but the space group selection rule (2.24) is not satisfied for any choice $h_{a} \in S$. This happens for example for the $\mathbb{Z}_{6}-\mathrm{II}-1-1$ orbifold, where the charges under $D_{S}$ do not depend on $n_{1}$ and $n_{2}$, i.e. on the localization of a twisted string in the first two-torus (often called the $G_{2}$ torus), see table 1. However, the space group selection rule applied to the first two-torus still constrains the allowed interactions [28]. Consequently, in such a case the SG flavor symmetry $D_{S}$ is not equivalent to the space group selection rule. It remains unknown whether one can modify our assumptions eqs. (3.1) and (3.2) such that the resulting discrete symmetry is fully equivalent to the space group selection rule.

\subsection{Consequences of the conditions}

Since $s$ is a representation of the space group $S$ we easily see that

$$
s\left(\mathbb{1}_{S}\right) s\left(\mathbb{1}_{S}\right)=s\left(\mathbb{1}_{S} \mathbb{1}_{S}\right)=s\left(\mathbb{1}_{S}\right) \Rightarrow s\left(\mathbb{1}_{S}\right)=\mathbb{1}_{D_{S}},
$$

where $\mathbb{1}_{D_{S}}$ is the identity element of $D_{S}$. Another consequence of $s$ being a representation of $S$ reads

$$
\mathbb{1}_{D_{S}}=s\left(\mathbb{1}_{S}\right)=s\left(g^{-1} g\right)=s\left(g^{-1}\right) s(g) \Rightarrow s\left(g^{-1}\right)=s(g)^{-1},
$$

for all $g \in S$. In addition, eq. (3.1) yields

$$
s(h g)=s(g h) \Leftrightarrow[s(h), s(g)]=0,
$$


for all $h, g \in S$. Thus, the SG flavor symmetry $D_{S}$ must be Abelian and the representation $s$ is in general not faithful. Furthermore, eqs. (3.6) and (3.7) yield the important condition

$$
s([g, h])=\mathbb{1}_{D_{S}},
$$

where the commutator is defined as $[g, h]=g^{-1} h^{-1} g h$, see section 2.2. Consequently, the representation $s$ of the space group $S$ is equivalent to the Abelianization of the space group $S$,

$$
D_{S} \cong S /[S, S]
$$

see e.g. ref. [29]. ${ }^{1}$

Indeed, it turns out that $D_{S}$ is a finite Abelian group for all space groups $S$ under consideration (i.e. in all cases the Abelian group $D_{S}$ does not contain any factors of $\mathbb{Z}$ ). Hence, we can represent $s(g)$ for all $g \in S$ by a complex phase,

$$
s(g)=\exp (\mathrm{i} \alpha(g)),
$$

and set $\mathbb{1}_{D_{S}}=1$ in the following. In addition, $D_{S}$ is a direct product of $\mathbb{Z}_{M_{i}}$ factors of various orders $M_{i}$.

In order to obtain the discrete transformation $s(g)$ for a string state $|[g]\rangle$ with constructing element $g \in S$ explicitly, we first express $g$ as a product of the generators of $S$, see section 2.1. In detail, in the case of a $\mathbb{Z}_{M} \times \mathbb{Z}_{N}$ point group the generators of $S$ read

$$
T_{i}=\left(\mathbb{1}, e_{i}\right), g_{\theta}=\left(\theta, \lambda_{\theta}\right) \text { and } g_{\omega}=\left(\omega, \lambda_{\omega}\right),
$$

such that we can decompose any space group element $g \in S$ as

$$
g=\left(T_{1}\right)^{n_{1}} \ldots\left(T_{D}\right)^{n_{D}}\left(g_{\theta}\right)^{k}\left(g_{\omega}\right)^{\ell}
$$

where $k=0, \ldots, M-1, \ell=0, \ldots, N-1$ and $n_{i} \in \mathbb{Z}$ for $i=1, \ldots, D$. Then, using the representation property eq. (3.2), we obtain

$$
s(g)=\left(s\left(T_{1}\right)\right)^{n_{1}} \ldots\left(s\left(T_{D}\right)\right)^{n_{D}}\left(s\left(g_{\theta}\right)\right)^{k}\left(s\left(g_{\omega}\right)\right)^{\ell} .
$$

Thus, we can easily compute the discrete transformation $s(g)$ of a string state $|[g]\rangle$ once we know the discrete transformations of the generators of the space group

$$
s\left(T_{i}\right), s\left(g_{\theta}\right) \text { and } s\left(g_{\omega}\right) .
$$

Hence, we have reduced our problem to the task of identifying these building-blocks of the general discrete transformation $s(g)$.

\footnotetext{
${ }^{1}$ The Abelianization of space groups was also used in ref. [30] to constrain the gauge embeddings via shifts and Wilson lines, and in relation to Gauged Linear Sigma Models (see also [31]).
} 


\subsection{Space group selection rule and the Abelianization of the space group}

As noted before, our conditions eqs. (3.1) and (3.2) that define the transformation $s(g)$ of a closed string state $|[g]\rangle$ with constructing element $g \in S$ correspond to the Abelianization of the space group $S$. In this section we show that the Abelianization of $S$ can be computed using the so-called presentation of the space group $S$. To do so, each generator $g$ of $S$ is replaced by $s(g)$ and each relation in the presentation of $S$ is replaced by $s$ (relation) using that $s([g, h])=1$, i.e.

$$
\begin{aligned}
g_{\theta}=\left(\theta, \lambda_{\theta}\right) & \longmapsto s\left(g_{\theta}\right), \\
g_{\omega}=\left(\omega, \lambda_{\omega}\right) & \longmapsto s\left(g_{\omega}\right), \\
T_{i}=\left(\mathbb{1}, e_{i}\right) & \longmapsto s\left(T_{i}\right), \quad i=1, \ldots, D,
\end{aligned}
$$

and from the presentation

$$
\left.S=\left\langle g_{\theta}, g_{\omega}, T_{1}, \ldots, T_{D}\right| g_{\theta}^{M}\left(T_{1}\right)^{-a_{(\theta, 1)}} \ldots\left(T_{D}\right)^{-a_{(\theta, D)}}, \ldots, \text { all relations }\right\rangle,
$$

see section 2.2 , we obtain via the map $S \mapsto D_{S}$ the presentation of $D_{S}$ as

$$
\begin{aligned}
D_{S}= & \left\langle s\left(g_{\theta}\right), s\left(g_{\omega}\right), s\left(T_{1}\right), \ldots, s\left(T_{D}\right)\right| \\
& \left.s\left(g_{\theta}\right)^{M}\left(s\left(T_{1}\right)\right)^{-a_{(\theta, 1)}} \ldots\left(s\left(T_{D}\right)\right)^{-a_{(\theta, D)}}, \ldots, s(\text { all relations })\right\rangle .
\end{aligned}
$$

In most cases, some generators $s(g)$ in the presentation of $D_{S}$ are no longer independent compared to their preimages $g \in S$. Thus, one has to solve the relations in $D_{S}$ such that only the independent generators remain.

Furthermore, for all space groups under consideration all remaining relations in $D_{S}$ can be solved explicitly such that one can identify the SG flavor symmetry $D_{S}$ as the direct product of cyclic groups, i.e.

$$
D_{S} \cong \mathbb{Z}_{M_{1}} \times \mathbb{Z}_{M_{2}} \times \cdots
$$

In the following we present two approaches to how this computation can be performed in detail.

\subsection{Direct computation}

Among the relations in the presentation of $D_{S}$ in eq. (3.17) there are two sets of relations that are of special interest: eqs. (2.15e) and (2.15f) (or equivalently eq. (2.9)) are related to the charges of translations $s\left(T_{i}\right)$, while eqs. $(2.15 \mathrm{a})$ and $(2.15 \mathrm{~b})$ are related to the charges of rotations $s\left(g_{\theta}\right)$ and $s\left(g_{\omega}\right)$. Using these relations one can easily compute the orders of $s\left(g_{\theta}\right), s\left(g_{\omega}\right)$ and $s\left(T_{i}\right)$, as we do next.

The charges of translations $s\left(\boldsymbol{T}_{\boldsymbol{i}}\right)$. We embed eq. (2.9) into $D_{S}$ and obtain ${ }^{2}$

$$
s\left(\mathbb{1}, e_{i}\right)=s\left(\mathbb{1}, \rho e_{i}\right),
$$

\footnotetext{
${ }^{2}$ For a space group element $g=(\rho, \lambda) \in S$ we write $s(g)=s(\rho, \lambda)$ instead of $s(g)=s((\rho, \lambda))$.
} 
for all $\rho \in P$. In other words, two vectors $e_{i}$ and $\rho e_{i}$ that are rotated to each other by a point group element $\rho$ give rise to the same element of $D_{S}$.

Now, we assume that there is a point group element $\rho \in P$ and a smallest integer $M_{i}$ such that

$$
e_{i}+\rho e_{i}+\ldots+\rho^{M_{i}-1} e_{i}=0 .
$$

We can translate this equation into space group elements of pure translations and apply $s$. This yields

$$
\underbrace{s\left(\mathbb{1}, e_{i}\right) s\left(\mathbb{1}, \rho e_{i}\right) \ldots s\left(\mathbb{1}, \rho^{M_{i}-1} e_{i}\right)}_{M_{i} \text { factors }}=1 .
$$

Then, using eq. (3.19) we get

$$
s\left(\mathbb{1}, e_{i}\right)^{M_{i}}=1 .
$$

Consequently, $s\left(T_{i}\right)=s\left(\mathbb{1}, e_{i}\right)$ generates a finite Abelian group of order $M_{i}$, being $\mathbb{Z}_{M_{i}}$, and we can express $s\left(T_{i}\right)$ as a complex phase

$$
s\left(T_{i}\right)=\exp \left(\frac{2 \pi \mathrm{i}}{M_{i}} \beta_{i}\right),
$$

for $\beta_{i} \in \mathbb{Z}$.

The charges of rotations $s\left(g_{\theta}\right)$ and $s\left(g_{\omega}\right)$. For example, consider the generator $\theta \in P$ of order $M$ in the case without roto-translations, i.e. $g_{\theta}=(\theta, 0)$. Then, we obtain the following identities

$$
s(\theta, 0)^{M}=s\left(\theta^{M}, 0\right)=s\left(\mathbb{1}_{S}\right)=1 .
$$

Consequently, $s\left(g_{\theta}\right)=s(\theta, 0)$ generates a finite Abelian group of order $M$, being $\mathbb{Z}_{M}$, and we can express $s\left(g_{\theta}\right)$ as a complex phase

$$
s\left(g_{\theta}\right)=\exp \left(\frac{2 \pi \mathrm{i}}{M} \alpha_{\theta}\right),
$$

for $\alpha_{\theta} \in \mathbb{Z}$. Analogously, using $\omega^{N}=\mathbb{1}$ we find

$$
s\left(g_{\omega}\right)=\exp \left(\frac{2 \pi \mathrm{i}}{N} \alpha_{\omega}\right)
$$

for $\alpha_{\omega} \in \mathbb{Z}$.

Combination of translations and rotations. As we have seen in eq. (3.13) the representation $s(g)$ of a general space group element eq. (3.12) is given by

$$
s(g)=\left(s\left(T_{1}\right)\right)^{n_{1}} \ldots\left(s\left(T_{D}\right)\right)^{n_{D}}\left(s\left(g_{\theta}\right)\right)^{k}\left(s\left(g_{\omega}\right)\right)^{\ell},
$$

where one might be tempted to simply replace the previous results for the representations of the translations, $s\left(T_{i}\right)$, and roto-translations, $s\left(g_{\theta}\right), s\left(g_{\omega}\right)$. However, in general, the presentation of $D_{S}$ eq. (3.17) establishes non-trivial relations among different elements, that can alter the result. 
In fact, only in the simplest case without roto-translations and without non-trivial relations between translations and twists, this naïve expectation holds. In this case, a general space group element simplifies to $g=\left(\theta^{k} \omega^{\ell}, n_{i} e_{i}\right) \in S$ and its representation is given by

$$
s:\left(\theta^{k} \omega^{\ell}, n_{i} e_{i}\right) \rightarrow \exp \left(2 \pi \mathrm{i}\left(\frac{k}{M} \alpha_{\theta}+\frac{\ell}{N} \alpha_{\omega}+\sum_{i=1}^{D} \frac{n_{i}}{M_{i}} \beta_{j}\right)\right),
$$

where $k, \ell$ and $n_{i}$ for $i=1, \ldots, D$ are discrete charges. They are conserved in an allowed coupling, i.e.

$$
\sum_{a=1}^{L} k^{(a)}=0 \bmod M, \quad \sum_{a=1}^{L} \ell^{(a)}=0 \bmod N, \quad \sum_{a=1}^{L} n_{i}^{(a)}=0 \bmod M_{i},
$$

where $k^{(a)}, \ell^{(a)}$ and $n_{i}^{(a)}$ define the $a$-th constructing element $g_{a} \in S$ in the coupling (2.22). As a remark, those symmetries that constrain the twisted sectors $k^{(a)}$ (and $\ell^{(a)}$ ) are conventionally referred to as point group selection rule (PG).

In the more general case, $\alpha_{\theta}, \alpha_{\omega}, \beta_{i}$ and $\beta_{j}$, with $i \neq j$, turn out to be connected by the relations in the presentation of $D_{S}$. These relations as well as their implications shall be discussed in our examples, in section 3.6.

\subsection{Space group selection rule and remnant discrete symmetries from spon- taneous symmetry breaking}

As before, we begin with a space group $S$ generated by $D+2$ generators

$$
T_{i}=\left(\mathbb{1}, e_{i}\right), i=1, \ldots, D, g_{\theta}=\left(\theta, \lambda_{\theta}\right) \text { and } g_{\omega}=\left(\omega, \lambda_{\omega}\right)
$$

subject to $K$ relations as listed in the presentation of $S$. Then, the SG flavor symmetry $D_{S}$ obtained from the space group selection rule is a subgroup of $\mathrm{U}(1)^{D+2}$. It can be computed as follows: each relation $\alpha=1, \ldots, K$ of the presentation of $S$ is mapped to a relation in $D_{S}$ and can be written in the form

$$
s\left(T_{1}\right)^{q_{1}^{\alpha}} \ldots s\left(T_{D}\right)^{q_{D}^{\alpha}} s\left(g_{\theta}\right)^{q_{D+1}^{\alpha}} s\left(g_{\omega}\right)^{q_{D+2}^{\alpha}}=1,
$$

see eq. (2.15) in section 2.2 using $s([g, h])=1$. To each relation (3.31) one can associate an auxiliary field $\phi_{\alpha}(x)$ with integer $\mathrm{U}(1)^{D+2}$ charges given by the exponents in eq. (3.31), i.e. the charges of $\phi_{\alpha}(x)$ read

$$
\left(q_{1}^{\alpha}, \ldots, q_{D}^{\alpha}, q_{D+1}^{\alpha}, q_{D+2}^{\alpha}\right)
$$

These auxiliary fields have no physical interpretation but are only used to describe the symmetry breaking associated to the relations (3.31). Then, under a general $\mathrm{U}(1)^{D+2}$ transformation the auxiliary field $\phi_{\alpha}(x)$ picks up a phase, i.e.

$$
\phi_{\alpha}(x) \mapsto \exp \left(\mathrm{i} \sum_{j=1}^{D+2} \xi_{j} q_{j}^{\alpha}\right) \phi_{\alpha}(x)
$$


where $\xi_{j} \in \mathbb{R}, j=1, \ldots, D+2$, denote the $\mathrm{U}(1)$ transformation parameters. Now, we turn on the vacuum expectation values (VEVs) of the auxiliary fields $\left\langle\phi_{\alpha}\right\rangle \neq 0$ for all $\alpha=1, \ldots, K$. Consequently, the unbroken remnant symmetry is given by the solutions $\xi_{j}$ of

$$
\exp \left(\mathrm{i} \sum_{j=1}^{D+2} \xi_{j} q_{j}^{\alpha}\right)\left\langle\phi_{\alpha}\right\rangle=\left\langle\phi_{\alpha}\right\rangle
$$

for all auxiliary fields $\alpha=1, \ldots, K$. Identifying

$$
s\left(T_{i}\right)=\exp \left(\mathrm{i} \xi_{i}\right), s\left(g_{\theta}\right)=\exp \left(\mathrm{i} \xi_{D+1}\right) \quad \text { and } s\left(g_{\omega}\right)=\exp \left(\mathrm{i} \xi_{D+2}\right),
$$

we realize that this spontaneous $\mathrm{U}(1)$ symmetry breaking exactly corresponds to solving the relations (3.31).

This method to compute the SG flavor symmetry as a remnant discrete symmetry via spontaneous symmetry breaking of $\mathrm{U}(1)^{D+2}$ can be automatized easily using for example the mathematica package "DiscreteBreaking" developed in ref. [32].

\subsection{Examples}

In this section we present three examples how to compute the SG flavor symmetries explicitly: first, we consider a $\mathbb{Z}_{3}$ toy example in $D=2$ dimensions and then two $\mathbb{Z}_{2} \times \mathbb{Z}_{2}$ examples in $D=6$ dimensions, one with roto-translations and the other with a freely-acting shift.

\subsection{1 $\mathbb{Z}_{3}$ space group in $D=2$ dimensions}

We define the two-dimensional $\mathbb{Z}_{3}$ space group $S$ by the generators

$$
T_{1}=\left(\mathbb{1}, e_{1}\right), \quad T_{2}=\left(\mathbb{1}, e_{2}\right) \text { and } g_{\theta}=(\theta, 0) \text {, }
$$

where the basis vectors $e_{1}$ and $e_{2}$ enclose an angle of $120^{\circ}$ and have equal length. Furthermore, $\theta$ is a counter-clockwise rotation by $120^{\circ}$ such that

$$
\theta e=e \hat{\theta} \quad \text { where } \quad \hat{\theta}=\left(\begin{array}{l}
0-1 \\
1-1
\end{array}\right) .
$$

This space group can be defined alternatively by the following abstract presentation, based on the three generators $g_{\theta}, T_{1}$ and $T_{2}$ subject to four relations, i.e.

$$
S=\left\langle g_{\theta}, T_{1}, T_{2} \mid g_{\theta}^{3},\left[T_{1}, T_{2}\right],\left[g_{\theta}, T_{1}\right] T_{1}^{-2} T_{2}^{-1},\left[g_{\theta}, T_{2}\right] T_{1} T_{2}^{-1}\right\rangle,
$$

see section 2.2 .

To compute the SG flavor symmetry $D_{S}$ we map the three generators of $S$ to $s\left(g_{\theta}\right)$, $s\left(T_{1}\right)$ and $s\left(T_{2}\right)$. Then, the presentation of $D_{S}$ reads

$$
\begin{aligned}
D_{S}= & \left\langle s\left(g_{\theta}\right), s\left(T_{1}\right), s\left(T_{2}\right)\right| s\left(g_{\theta}\right)^{3}, \underbrace{s\left(\left[T_{1}, T_{2}\right]\right)}_{=1}, \underbrace{s\left(\left[g_{\theta}, T_{1}\right]\right)}_{=1} s\left(T_{1}\right)^{-2} s\left(T_{2}\right)^{-1}, \\
& \underbrace{s\left(\left[g_{\theta}, T_{2}\right]\right)}_{=1} s\left(T_{1}\right) s\left(T_{2}\right)^{-1}\rangle .
\end{aligned}
$$


Next, we omit the trivial relation $s\left(\left[T_{1}, T_{2}\right]\right)=1$ and obtain

$$
D_{S}=\left\langle s\left(g_{\theta}\right), s\left(T_{1}\right), s\left(T_{2}\right) \mid s\left(g_{\theta}\right)^{3}, s\left(T_{1}\right)^{-2} s\left(T_{2}\right)^{-1}, s\left(T_{1}\right) s\left(T_{2}\right)^{-1}\right\rangle .
$$

In the final step, we observe that only two of the three generators of $D_{S}$ are independent. For example, we can use the last relation $s\left(T_{1}\right) s\left(T_{2}\right)^{-1}=1$ in eq. (3.40) to replace $s\left(T_{2}\right)$ by $s\left(T_{1}\right)$ using $s\left(T_{2}\right)=s\left(T_{1}\right)$. Thus, we get

$$
D_{S}=\left\langle s\left(g_{\theta}\right), s\left(T_{1}\right) \mid s\left(g_{\theta}\right)^{3}, s\left(T_{1}\right)^{3}\right\rangle \cong \mathbb{Z}_{3} \times \mathbb{Z}_{3},
$$

and a general string state $|[g]\rangle$ transforms as

$$
\begin{aligned}
& |[g]\rangle \longmapsto s\left(T_{1}\right)^{n_{1}} s\left(T_{2}\right)^{n_{2}} s\left(g_{\theta}\right)^{k}|[g]\rangle=s\left(T_{1}\right)^{n_{1}+n_{2}} s\left(g_{\theta}\right)^{k}|[g]\rangle \\
& =\exp \left(\frac{2 \pi \mathrm{i}}{3} \alpha_{1}\left(n_{1}+n_{2}\right)\right) \exp \left(\frac{2 \pi \mathrm{i}}{3} \alpha_{\theta} k\right)|[g]\rangle,
\end{aligned}
$$

for $\alpha_{1}, \alpha_{\theta} \in\{0,1,2\}$ using $s\left(g_{\theta}\right)^{3}=s\left(T_{1}\right)^{3}=1$. Then, a coupling $\left|\left[g_{1}\right]\right\rangle \ldots\left|\left[g_{L}\right]\right\rangle$ of string states $\left|\left[g_{a}\right]\right\rangle$ with constructing elements $g_{a}=\left(\theta^{k^{(a)}}, n_{1}^{(a)} e_{1}+n_{2}^{(a)} e_{2}\right)$ is allowed by the SG flavor symmetry $D_{S} \cong \mathbb{Z}_{3} \times \mathbb{Z}_{3}$ if

$$
\sum_{a=1}^{L} k^{(a)}=0 \bmod 3 \text { and } \sum_{a=1}^{L}\left(n_{1}^{(a)}+n_{2}^{(a)}\right)=0 \bmod 3,
$$

where the first equation in eq. (3.44) is called point group selection rule (PG).

In summary, the space group selection rule of the two-dimensional $\mathbb{Z}_{3}$ space group yields a $\mathbb{Z}_{3} \times \mathbb{Z}_{3}$ SG flavor symmetry with discrete charges $k$ and $n_{1}+n_{2}$, respectively [20].

\subsubsection{Space group with freely-acting shift: $\mathbb{Z}_{2} \times \mathbb{Z}_{2}-5-1$}

Consider the so-called Blaszczyk-geometry $\mathbb{Z}_{2} \times \mathbb{Z}_{2}-5-1$ [25, 26] (in ref. [33] it is labeled as 1-1, see also [34], and [35] for an MSSM-like orbifold model based on this geometry using a different convention). The twists in the lattice basis (see eq. (2.10)) read

$$
\hat{\theta}=\left(\begin{array}{cccccc}
0 & 1 & -1 & 0 & 0 & 0 \\
1 & 0 & -1 & 0 & 0 & 0 \\
0 & 0 & -1 & 0 & 0 & 0 \\
0 & 0 & 0 & 1 & 0 & 0 \\
0 & 0 & 0 & 0 & -1 & 0 \\
0 & 0 & 0 & 0 & 0 & -1
\end{array}\right) \text { and } \hat{\omega}=\left(\begin{array}{cccccc}
0 & -1 & 1 & 0 & 0 & 0 \\
0 & -1 & 0 & 0 & 0 & 0 \\
1 & -1 & 0 & 0 & 0 & 0 \\
0 & 0 & 0 & -1 & 0 & 0 \\
0 & 0 & 0 & 0 & 1 & 0 \\
0 & 0 & 0 & 0 & 0 & -1
\end{array}\right) .
$$

The space group $S$ is generated by six translations $T_{i}=\left(\mathbb{1}, e_{i}\right)$ and two rotations

$$
g_{\theta}=(\theta, 0) \text { and } g_{\omega}=(\omega, 0) .
$$

By writing down the presentation of this space group $S$ we identify the relations

$$
\begin{gathered}
s\left(T_{1}\right)=s\left(T_{2}\right)=s\left(T_{3}\right) \quad \text { where } s\left(T_{1}\right)^{2} s\left(T_{2}\right) s\left(T_{3}\right)=1, \\
s\left(T_{4}\right)^{2}=s\left(T_{5}\right)^{2}=s\left(T_{6}\right)^{2}=s\left(g_{\theta}\right)^{2}=s\left(g_{\omega}\right)^{2}=1,
\end{gathered}
$$


see eq. (2.15). Consequently, the most general representation $s(g)$ is given by

$$
\begin{aligned}
s(g) & =s\left(T_{1}\right)^{n_{1}} s\left(T_{2}\right)^{n_{2}} s\left(T_{3}\right)^{n_{3}} s\left(T_{4}\right)^{n_{4}} s\left(T_{5}\right)^{n_{5}} s\left(T_{6}\right)^{n_{6}} s\left(g_{\theta}\right)^{k} s\left(g_{\omega}\right)^{\ell} \\
& =s\left(T_{1}\right)^{n_{1}+n_{2}+n_{3}} s\left(T_{4}\right)^{n_{4}} s\left(T_{5}\right)^{n_{5}} s\left(T_{6}\right)^{n_{6}} s\left(g_{\theta}\right)^{k} s\left(g_{\omega}\right)^{\ell},
\end{aligned}
$$

where we have used $s\left(T_{1}\right)=s\left(T_{2}\right)=s\left(T_{3}\right)$. The order of the basic transformations $s\left(g_{\theta}\right)$, $s\left(g_{\omega}\right), s\left(T_{4}\right), s\left(T_{5}\right)$ and $s\left(T_{6}\right)$ is 2 . Furthermore, from eq. (3.47a) we obtain

$$
s\left(T_{1}\right)^{2} s\left(T_{2}\right) s\left(T_{3}\right)=s\left(T_{1}\right)^{4}=1,
$$

and $s\left(T_{1}\right)$ generates a $\mathbb{Z}_{4}$ factor. Consequently, all transformations $s\left(g_{\theta}\right), s\left(g_{\omega}\right), s\left(T_{1}\right)$, $s\left(T_{4}\right), s\left(T_{5}\right)$ and $s\left(T_{6}\right)$ are independent. Hence, the SG flavor symmetry $D_{S}$ obtained from the space group selection rule is given by

$$
\left(\mathbb{Z}_{2} \times \mathbb{Z}_{2}\right)^{\mathrm{PG}} \times \mathbb{Z}_{4} \times \mathbb{Z}_{2} \times \mathbb{Z}_{2} \times \mathbb{Z}_{2},
$$

with discrete charges

$$
k, \ell, n_{1}+n_{2}+n_{3}, n_{4}, n_{5} \text { and } n_{6},
$$

respectively, and we have verified this result additionally using the VEV-method of section 3.5.

$\mathbb{Z}_{\mathbf{2}}$ dark matter-parity. By considering the fixed points of this $\mathbb{Z}_{2} \times \mathbb{Z}_{2}$ orbifold, one can check that all massless strings carry even $\mathbb{Z}_{4}$ charges, i.e.

$$
n_{1}+n_{2}+n_{3} \in\{0,2\} .
$$

Hence, the allowed interactions of massless strings are not constrained by a $\mathbb{Z}_{4}$ factor in eq. (3.50), but only by $\mathbb{Z}_{2}$. However, there are massive strings with odd discrete charges $n_{1}+n_{2}+n_{3} \in\{1,3\}$ under $\mathbb{Z}_{4}$, for example a winded string with constructing element $\left(\mathbb{1}, e_{1}\right)$. Consequently, massive strings with odd $\mathbb{Z}_{4}$ charges can only be produced and annihilated in pairs. Thus, the lightest massive string with odd $\mathbb{Z}_{4}$ charge could serve as a dark matter candidate, which is stable because there is a $\mathbb{Z}_{2}$ dark matter-parity with

$$
\begin{aligned}
\mid \text { matter }\rangle & \longmapsto+\mid \text { matter }\rangle, \\
\mid \text { dark matter }\rangle & \longmapsto-\mid \text { dark matter }\rangle,
\end{aligned}
$$

and the mass of the dark matter particle depends on the compactification radii. This fact is common to many SG flavor symmetries in table 1 and might be relevant for the observed dark matter content of the universe and also for its cosmological evolution.

\subsubsection{Space group with roto-translation: $\mathbb{Z}_{2} \times \mathbb{Z}_{2}-2-5$}

Consider the $\mathbb{Z}_{2} \times \mathbb{Z}_{2}-2-5$ orbifold from the classification in refs. [25, 26] (it corresponds to the $\mathbb{Z}_{2} \times \mathbb{Z}_{2}$ orbifold labeled 1-9 in ref. [33]). The twists in the lattice basis (see eq. (2.10)) 
are given by

$$
\hat{\theta}=\left(\begin{array}{cccccc}
0 & 1 & 0 & 0 & 0 & 0 \\
1 & 0 & 0 & 0 & 0 & 0 \\
0 & 0 & 1 & 0 & 0 & 0 \\
0 & 0 & 0 & -1 & 0 & 0 \\
0 & 0 & 0 & 0 & -1 & 0 \\
0 & 0 & 0 & 0 & 0 & -1
\end{array}\right) \text { and } \hat{\omega}=\left(\begin{array}{cccccc}
-1 & 0 & 0 & 0 & 0 & 0 \\
0 & -1 & 0 & 0 & 0 & 0 \\
0 & 0 & -1 & 0 & 0 & 0 \\
0 & 0 & 0 & 1 & 0 & 0 \\
0 & 0 & 0 & 0 & 1 & 0 \\
0 & 0 & 0 & 0 & 0 & -1
\end{array}\right) .
$$

The space group $S$ is generated by six translations $T_{i}=\left(\mathbb{1}, e_{i}\right)$ and two roto-translations

$$
g_{\theta}=\left(\theta, \frac{1}{2} e_{3}\right) \quad \text { and } \quad g_{\omega}=\left(\omega, \frac{1}{2} e_{5}\right) .
$$

By writing down the presentation of $S$ we identify the relations (see eq. (2.15))

$$
\begin{gathered}
s\left(T_{1}\right)=s\left(T_{2}\right), s\left(T_{3}\right)=s\left(T_{5}\right)=s\left(g_{\theta}\right)^{2}=s\left(g_{\omega}\right)^{2} \text { and } \\
s\left(T_{i}\right)^{2}=s\left(g_{\theta}\right)^{4}=s\left(g_{\omega}\right)^{4}=1 \text { for } i=1, \ldots, 6
\end{gathered}
$$

Consequently, the most general charge $s(g)$ is given by

$$
\begin{aligned}
s(g) & =s\left(T_{1}\right)^{n_{1}} s\left(T_{2}\right)^{n_{2}} s\left(T_{3}\right)^{n_{3}} s\left(T_{4}\right)^{n_{4}} s\left(T_{5}\right)^{n_{5}} s\left(T_{6}\right)^{n_{6}} s\left(g_{\theta}\right)^{k} s\left(g_{\omega}\right)^{\ell} \\
& =s\left(T_{1}\right)^{n_{1}+n_{2}} s\left(T_{4}\right)^{n_{4}} s\left(T_{6}\right)^{n_{6}} s\left(g_{\theta}\right)^{k+2\left(n_{3}+n_{5}\right)} s\left(g_{\omega}\right)^{\ell},
\end{aligned}
$$

where we have used $s\left(T_{1}\right)=s\left(T_{2}\right)$ and $s\left(T_{3}\right)=s\left(T_{5}\right)=s\left(g_{\theta}\right)^{2}$. Next, we analyze the consequences of $s\left(g_{\theta}\right)^{2}=s\left(g_{\omega}\right)^{2}$ from eq. (3.56a), i.e. we make the ansatz

$$
s\left(g_{\theta}\right)=\exp \left(\frac{2 \pi \mathrm{i}}{4} \alpha_{\theta}\right) \text { and } s\left(g_{\omega}\right)=\exp \left(\frac{2 \pi \mathrm{i}}{4} \alpha_{\omega}\right) .
$$

Then, $s\left(g_{\theta}\right)^{2}=s\left(g_{\omega}\right)^{2}$ yields

$$
\exp \left(\frac{2 \pi \mathrm{i}}{2} \alpha_{\theta}\right)=\exp \left(\frac{2 \pi \mathrm{i}}{2} \alpha_{\omega}\right) \Leftrightarrow \alpha_{\omega}=\alpha_{\theta}+2 x
$$

for some $x \in \mathbb{Z}$. Thus,

$$
s\left(g_{\omega}\right)=\exp \left(\frac{2 \pi \mathrm{i}}{4} \alpha_{\omega}\right)=\exp \left(\frac{2 \pi \mathrm{i}}{4} \alpha_{\theta}\right) \exp \left(\frac{2 \pi \mathrm{i}}{2} x\right)=s\left(g_{\theta}\right) s_{x} .
$$

where $s\left(g_{\theta}\right)$ and $s_{x}$ are now independent and of order 4 and 2 , respectively. Using eq. (3.60) in eq. (3.57b) we obtain

$$
s(g)=s\left(g_{\theta}\right)^{k+\ell+2\left(n_{3}+n_{5}\right)} s_{x}^{\ell} s\left(T_{1}\right)^{n_{1}+n_{2}} s\left(T_{4}\right)^{n_{4}} s\left(T_{6}\right)^{n_{6}},
$$

where the orders of $s\left(g_{\theta}\right), s_{x}, s\left(T_{1}\right), s\left(T_{4}\right)$ and $s\left(T_{6}\right)$ are 4, 2, 2, 2 and 2, respectively. Now, we have solved all relations (3.56) and, consequently, all transformations $s\left(g_{\theta}\right), s_{x}, s\left(T_{1}\right)$, 
$s\left(T_{4}\right)$ and $s\left(T_{6}\right)$ are independent. Hence, the space group selection rule results in a SG flavor symmetry

$$
\left(\mathbb{Z}_{4} \times \mathbb{Z}_{2}\right)^{\mathrm{PG}} \times \mathbb{Z}_{2} \times \mathbb{Z}_{2} \times \mathbb{Z}_{2}
$$

with discrete charges

$$
k+\ell+2\left(n_{3}+n_{5}\right), \ell, n_{1}+n_{2}, n_{4} \text { and } n_{6},
$$

respectively. This result has also been verified using the VEV-method of section 3.5.

Naively, one would expect a $\mathbb{Z}_{2} \times \mathbb{Z}_{2}$ point group selection rule with charges $k$ and $\ell$ if the point group is $\mathbb{Z}_{2} \times \mathbb{Z}_{2}$. However, we have seen that the point group selection rule yields $\mathbb{Z}_{4} \times \mathbb{Z}_{2}$ with charges $k+\ell+2\left(n_{3}+n_{5}\right)$ and $\ell$. If one considers the $\mathbb{Z}_{2}$ subgroup of the $\mathbb{Z}_{4}$ factor, one identifies the corresponding $\mathbb{Z}_{2}$ charges as $k+\ell$. Thus, the naive $\mathbb{Z}_{2} \times \mathbb{Z}_{2}$ point group selection rule is a subgroup of the full $\mathbb{Z}_{4} \times \mathbb{Z}_{2}$ point group selection rule.

\section{Results}

In this section we present the main result of this work: we compute the Abelian SG flavor symmetries $D_{S}$ for all 138 space groups $S$ with Abelian point group and $\mathcal{N}=1$ supersymmetry. To do so, we adopt the convention of refs. [25, 26] as specified in the geometry files

\begin{tabular}{|c|c|c|c|}
\hline $\begin{array}{l}\text { Q-class } \\
\text { twist vector }\end{array}$ & $\begin{array}{c}\mathbb{Z} \text { - and } \\
\text { affine class }\end{array}$ & $\begin{array}{l}\text { SG flavor } \\
\text { symmetry } D_{S}\end{array}$ & $\begin{array}{l}\text { discrete } \\
\text { charge }\end{array}$ \\
\hline $\begin{array}{c}\mathbb{Z}_{3} \\
\left(0, \frac{1}{3}, \frac{1}{3},-\frac{2}{3}\right)\end{array}$ & $1-1$ & $\begin{array}{l}\mathbb{Z}_{3}^{(\mathrm{PG})} \\
\left(\mathbb{Z}_{3}\right)^{3} \\
\end{array}$ & $\begin{array}{l}k \\
\left(n_{1}+n_{2}, n_{3}+n_{4}, n_{5}+n_{6}\right)\end{array}$ \\
\hline \multirow[t]{3}{*}{$\begin{array}{c}\mathbb{Z}_{4} \\
\left(0, \frac{1}{4}, \frac{1}{4},-\frac{1}{2}\right)\end{array}$} & $1-1$ & $\begin{array}{l}\mathbb{Z}_{4}^{(\mathrm{PG})} \\
\left(\mathbb{Z}_{2}\right)^{4}\end{array}$ & $\begin{array}{l}k \\
\left(n_{1}+n_{2}, n_{3}+n_{4}, n_{5}, n_{6}\right)\end{array}$ \\
\hline & $2-1$ & $\begin{array}{l}\mathbb{Z}_{4}^{(\mathrm{PG})} \\
\mathbb{Z}_{4} \times\left(\mathbb{Z}_{2}\right)^{2}\end{array}$ & $\begin{array}{l}k \\
\left(n_{3}+n_{4}+n_{5}, n_{1}+n_{2}, n_{6}\right)\end{array}$ \\
\hline & $3-1$ & $\begin{array}{l}\mathbb{Z}_{4}^{(\mathrm{PG})} \\
\left(\mathbb{Z}_{4}\right)^{2}\end{array}$ & $\begin{array}{l}k \\
\left(n_{1}+n_{2}+n_{3}, n_{4}+n_{5}+n_{6}\right)\end{array}$ \\
\hline \multirow[t]{2}{*}{$\begin{array}{c}\mathbb{Z}_{6}-\mathrm{I} \\
\left(0, \frac{1}{6}, \frac{1}{6},-\frac{1}{3}\right)\end{array}$} & $1-1$ & $\begin{array}{l}\mathbb{Z}_{6}^{(\mathrm{PG})} \\
\mathbb{Z}_{3}\end{array}$ & $\begin{array}{l}k \\
n_{5}+n_{6}\end{array}$ \\
\hline & $2-1$ & $\begin{array}{l}\mathbb{Z}_{6}^{(\mathrm{PG})} \\
\mathbb{Z}_{3}\end{array}$ & $\begin{array}{l}k \\
n_{3}+n_{4}+n_{5}+n_{6}\end{array}$ \\
\hline \multirow[t]{3}{*}{$\begin{array}{c}\mathbb{Z}_{6}-\mathrm{II} \\
\left(0, \frac{1}{6}, \frac{1}{3},-\frac{1}{2}\right)\end{array}$} & $1-1$ & $\begin{array}{l}\mathbb{Z}_{6}^{(\mathrm{PG})} \\
\mathbb{Z}_{3} \times\left(\mathbb{Z}_{2}\right)^{2}\end{array}$ & $\begin{array}{l}k \\
\left(n_{3}+n_{4}, n_{5}, n_{6}\right) \\
\end{array}$ \\
\hline & $2-1$ & $\begin{array}{l}\mathbb{Z}_{6}^{(\mathrm{PG})} \\
\mathbb{Z}_{3} \times\left(\mathbb{Z}_{2}\right)^{2}\end{array}$ & $\begin{array}{l}k \\
\left(n_{1}+n_{2}+n_{3}+n_{4}, n_{5}, n_{6}\right)\end{array}$ \\
\hline & $3-1$ & $\begin{array}{l}\mathbb{Z}_{6}^{(\mathrm{PG})} \\
\mathbb{Z}_{3} \times\left(\mathbb{Z}_{2}\right)^{2}\end{array}$ & $\begin{array}{l}k \\
\left(n_{4}+n_{5}, n_{1}+n_{2}+n_{3}, n_{6}\right)\end{array}$ \\
\hline
\end{tabular}
for the orbifolder [36] (see ancillary files in arXiv.org). The results are listed in table 1.

continued... 


\begin{tabular}{|c|c|c|c|}
\hline $\begin{array}{l}\text { Q-class } \\
\text { twist vector }\end{array}$ & $\begin{array}{l}\mathbb{Z} \text { - and } \\
\text { affine class }\end{array}$ & $\begin{array}{l}\text { SG flavor } \\
\text { symmetry } D_{S}\end{array}$ & $\begin{array}{l}\text { discrete } \\
\text { charge }\end{array}$ \\
\hline & $4-1$ & $\begin{array}{l}\mathbb{Z}_{6}^{(\mathrm{PG})} \\
\mathbb{Z}_{6} \times \mathbb{Z}_{2}\end{array}$ & $\begin{array}{l}k \\
\left(n_{1}+n_{2}+n_{3}+n_{4}+n_{5}, n_{6}\right)\end{array}$ \\
\hline $\begin{array}{c}\mathbb{Z}_{7} \\
\left(0, \frac{1}{7}, \frac{2}{7},-\frac{3}{7}\right)\end{array}$ & $1-1$ & $\begin{array}{l}\mathbb{Z}_{7}^{(\mathrm{PG})} \\
\mathbb{Z}_{7}\end{array}$ & $\begin{array}{l}k \\
n_{1}+n_{2}+n_{3}+n_{4}+n_{5}+n_{6}\end{array}$ \\
\hline \multirow[t]{3}{*}{$\begin{array}{c}\mathbb{Z}_{8}-\mathrm{I} \\
\left(0, \frac{1}{8}, \frac{1}{4},-\frac{3}{8}\right)\end{array}$} & $1-1$ & $\begin{array}{l}\mathbb{Z}_{8}^{(\mathrm{PG})} \\
\left(\mathbb{Z}_{2}\right)^{2}\end{array}$ & $\begin{array}{l}k \\
\left(n_{1}+n_{2}+n_{3}+n_{4}, n_{5}+n_{6}\right)\end{array}$ \\
\hline & $2-1$ & $\begin{array}{l}\mathbb{Z}_{8}^{(\mathrm{PG})} \\
\left(\mathbb{Z}_{2}\right)^{2}\end{array}$ & $\begin{array}{l}k \\
\left(n_{1}+n_{2}+n_{3}+n_{4}, n_{5}+n_{6}\right)\end{array}$ \\
\hline & $3-1$ & $\begin{array}{l}\mathbb{Z}_{8}^{(\mathrm{PG})} \\
\mathbb{Z}_{4}\end{array}$ & $\begin{array}{l}k \\
n_{1}+n_{2}+n_{3}+n_{4}+n_{5}+n_{6}\end{array}$ \\
\hline \multirow[t]{2}{*}{$\begin{array}{c}\mathbb{Z}_{8}-\mathrm{II} \\
\left(0, \frac{1}{8}, \frac{3}{8},-\frac{1}{2}\right)\end{array}$} & $1-1$ & $\begin{array}{l}\mathbb{Z}_{8}^{(\mathrm{PG})} \\
\left(\mathbb{Z}_{2}\right)^{3} \\
\end{array}$ & $\begin{array}{l}k \\
\left(n_{1}+n_{2}+n_{3}+n_{4}, n_{5}, n_{6}\right)\end{array}$ \\
\hline & $2-1$ & $\begin{array}{l}\mathbb{Z}_{8}^{(\mathrm{PG})} \\
\mathbb{Z}_{4} \times \mathbb{Z}_{2}\end{array}$ & $\begin{array}{l}k \\
\left(n_{1}+n_{2}+n_{3}+n_{4}+n_{5}, n_{6}\right)\end{array}$ \\
\hline \multirow[t]{2}{*}{$\begin{array}{c}\mathbb{Z}_{12}-\mathrm{I} \\
\left(0, \frac{1}{12}, \frac{1}{3},-\frac{5}{12}\right)\end{array}$} & $1-1$ & $\begin{array}{l}\mathbb{Z}_{12}^{(\mathrm{PG})} \\
\mathbb{Z}_{3}\end{array}$ & $\begin{array}{l}k \\
n_{5}+n_{6}\end{array}$ \\
\hline & $2-1$ & $\begin{array}{l}\mathbb{Z}_{12}^{(\mathrm{PG})} \\
\mathbb{Z}_{3}\end{array}$ & $\begin{array}{l}k \\
n_{1}+n_{2}+n_{3}+n_{4}+n_{5}+n_{6}\end{array}$ \\
\hline $\begin{array}{c}\mathbb{Z}_{12}-\mathrm{II} \\
\left(0, \frac{1}{12}, \frac{5}{12},-\frac{1}{2}\right) \\
\end{array}$ & $1-1$ & $\begin{array}{l}\mathbb{Z}_{12}^{(\mathrm{PG})} \\
\left(\mathbb{Z}_{2}\right)^{2}\end{array}$ & $\begin{array}{l}k \\
\left(n_{5}, n_{6}\right) \\
\end{array}$ \\
\hline \multirow{8}{*}{$\begin{array}{c}\mathbb{Z}_{2} \times \mathbb{Z}_{2} \\
\left(0,0, \frac{1}{2},-\frac{1}{2}\right) \\
\left(0, \frac{1}{2}, 0,-\frac{1}{2}\right)\end{array}$} & $1-1$ & $\begin{array}{l}\left(\mathbb{Z}_{2} \times \mathbb{Z}_{2}\right)^{(\mathrm{PG})} \\
\left(\mathbb{Z}_{2}\right)^{6}\end{array}$ & $\begin{array}{l}(k, \ell) \\
\left(n_{1}, n_{2}, n_{3}, n_{4}, n_{5}, n_{6}\right)\end{array}$ \\
\hline & $1-2$ & $\begin{array}{l}\left(\mathbb{Z}_{2} \times \mathbb{Z}_{2}\right)^{(\mathrm{PG})} \\
\left(\mathbb{Z}_{2}\right)^{5}\end{array}$ & $\begin{array}{l}(k, \ell) \\
\left(n_{1}, n_{3}, n_{4}, n_{5}, n_{6}\right)\end{array}$ \\
\hline & $1-3$ & $\begin{array}{l}\left(\mathbb{Z}_{4} \times \mathbb{Z}_{2}\right)^{(\mathrm{PG})} \\
\left(\mathbb{Z}_{2}\right)^{4}\end{array}$ & $\begin{array}{l}\left(k+2\left(n_{2}+n_{6}\right), \ell\right) \\
\left(n_{1}, n_{3}, n_{4}, n_{5}\right)\end{array}$ \\
\hline & $1-4$ & $\begin{array}{l}\left(\mathbb{Z}_{4} \times \mathbb{Z}_{4}\right)^{(\mathrm{PG})} \\
\left(\mathbb{Z}_{2}\right)^{3}\end{array}$ & $\begin{array}{l}\left(k+2\left(n_{2}+n_{6}\right), \ell+2\left(n_{4}+n_{6}\right)\right) \\
\left(n_{1}, n_{3}, n_{5}\right)\end{array}$ \\
\hline & $2-1$ & $\begin{array}{l}\left(\mathbb{Z}_{2} \times \mathbb{Z}_{2}\right)^{(\mathrm{PG})} \\
\left(\mathbb{Z}_{2}\right)^{5}\end{array}$ & $\begin{array}{l}(k, \ell) \\
\left(n_{1}+n_{2}, n_{3}, n_{4}, n_{5}, n_{6}\right)\end{array}$ \\
\hline & $2-2$ & $\begin{array}{l}\left(\mathbb{Z}_{2} \times \mathbb{Z}_{2}\right)^{(\mathrm{PG})} \\
\left(\mathbb{Z}_{2}\right)^{4}\end{array}$ & $\begin{array}{l}(k, \ell) \\
\left(n_{1}+n_{2}, n_{4}, n_{5}, n_{6}\right)\end{array}$ \\
\hline & $2-3$ & $\begin{array}{l}\left(\mathbb{Z}_{4} \times \mathbb{Z}_{2}\right)^{(\mathrm{PG})} \\
\left(\mathbb{Z}_{2}\right)^{3}\end{array}$ & $\begin{array}{l}\left(k+2\left(n_{3}+n_{6}\right), \ell\right) \\
\left(n_{1}+n_{2}, n_{4}, n_{5}\right)\end{array}$ \\
\hline & $2-4$ & $\begin{array}{l}\left(\mathbb{Z}_{2} \times \mathbb{Z}_{2}\right)^{(\mathrm{PG})} \\
\left(\mathbb{Z}_{2}\right)^{4}\end{array}$ & $\begin{array}{l}(k, \ell) \\
\left(n_{1}+n_{2}, n_{3}, n_{4}, n_{6}\right)\end{array}$ \\
\hline
\end{tabular}

continued... 


\begin{tabular}{|c|c|c|c|}
\hline $\begin{array}{c}\text { Q-class } \\
\text { twist vector }\end{array}$ & $\begin{array}{l}\mathbb{Z} \text { - and } \\
\text { affine class }\end{array}$ & $\begin{array}{l}\text { SG flavor } \\
\text { symmetry } D_{S}\end{array}$ & $\begin{array}{l}\text { discrete } \\
\text { charge }\end{array}$ \\
\hline & $2-5$ & $\begin{array}{l}\left(\mathbb{Z}_{4} \times \mathbb{Z}_{2}\right)^{(\mathrm{PG})} \\
\left(\mathbb{Z}_{2}\right)^{3}\end{array}$ & $\begin{array}{l}\left(k+\ell+2\left(n_{3}+n_{5}\right), \ell\right) \\
\left(n_{1}+n_{2}, n_{4}, n_{6}\right)\end{array}$ \\
\hline & $2-6$ & $\begin{array}{l}\left(\mathbb{Z}_{4} \times \mathbb{Z}_{4}\right)^{(\mathrm{PG})} \\
\left(\mathbb{Z}_{2}\right)^{2}\end{array}$ & $\begin{array}{l}\left(k+2\left(n_{3}+n_{6}\right), \ell+2\left(n_{5}+n_{6}\right)\right) \\
\left(n_{1}+n_{2}, n_{4}\right)\end{array}$ \\
\hline & $3-1$ & $\begin{array}{l}\left(\mathbb{Z}_{2} \times \mathbb{Z}_{2}\right)^{(\mathrm{PG})} \\
\left(\mathbb{Z}_{2}\right)^{5}\end{array}$ & $\begin{array}{l}(k, \ell) \\
\left(n_{1}, n_{2}+n_{3}, n_{4}, n_{5}, n_{6}\right) \\
\end{array}$ \\
\hline & $3-2$ & $\begin{array}{l}\left(\mathbb{Z}_{2} \times \mathbb{Z}_{2}\right)^{(\mathrm{PG})} \\
\left(\mathbb{Z}_{2}\right)^{4}\end{array}$ & $\begin{array}{l}(k, \ell) \\
\left(n_{1}, n_{2}+n_{3}, n_{4}, n_{5}\right)\end{array}$ \\
\hline & $3-3$ & $\begin{array}{l}\left(\mathbb{Z}_{2} \times \mathbb{Z}_{4}\right)^{(\mathrm{PG})} \\
\left(\mathbb{Z}_{2}\right)^{3}\end{array}$ & $\begin{array}{l}\left(k, \ell+2\left(n_{5}+n_{6}\right)\right) \\
\left(n_{1}, n_{2}+n_{3}, n_{4}\right)\end{array}$ \\
\hline & $3-4$ & $\begin{array}{l}\left(\mathbb{Z}_{4} \times \mathbb{Z}_{4}\right)^{(\mathrm{PG})} \\
\left(\mathbb{Z}_{2}\right)^{2}\end{array}$ & $\begin{array}{l}\left(k+2\left(n_{4}+n_{6}\right), \ell+2\left(n_{5}+n_{6}\right)\right) \\
\left(n_{1}, n_{2}+n_{3}\right)\end{array}$ \\
\hline & $4-1$ & $\begin{array}{l}\left(\mathbb{Z}_{2} \times \mathbb{Z}_{2}\right)^{(\mathrm{PG})} \\
\left(\mathbb{Z}_{2}\right)^{4}\end{array}$ & $\begin{array}{l}(k, \ell) \\
\left(n_{1}+n_{2}, n_{3}, n_{4}, n_{5}+n_{6}\right)\end{array}$ \\
\hline & $4-2$ & $\begin{array}{l}\left(\mathbb{Z}_{2} \times \mathbb{Z}_{2}\right)^{(\mathrm{PG})} \\
\left(\mathbb{Z}_{2}\right)^{3}\end{array}$ & $\begin{array}{l}(k, \ell) \\
\left(n_{1}+n_{2}, n_{3}, n_{5}+n_{6}\right)\end{array}$ \\
\hline & $5-1$ & $\begin{array}{l}\left(\mathbb{Z}_{2} \times \mathbb{Z}_{2}\right)^{(\mathrm{PG})} \\
\mathbb{Z}_{4} \times\left(\mathbb{Z}_{2}\right)^{3}\end{array}$ & $\begin{array}{l}(k, \ell) \\
\left(n_{1}+n_{2}+n_{3}, n_{4}, n_{5}, n_{6}\right)\end{array}$ \\
\hline & $5-2$ & $\begin{array}{l}\left(\mathbb{Z}_{2} \times \mathbb{Z}_{2}\right)^{(\mathrm{PG})} \\
\mathbb{Z}_{4} \times\left(\mathbb{Z}_{2}\right)^{2}\end{array}$ & $\begin{array}{l}(k, \ell) \\
\left(n_{1}+n_{2}+n_{3}, n_{5}, n_{6}\right)\end{array}$ \\
\hline & $5-3$ & $\begin{array}{l}\left(\mathbb{Z}_{2} \times \mathbb{Z}_{2}\right)^{(\mathrm{PG})} \\
\left(\mathbb{Z}_{2}\right)^{4}\end{array}$ & $\begin{array}{l}(k, \ell) \\
\left(n_{1}+n_{2}+n_{3}, n_{4}, n_{5}, n_{6}\right)\end{array}$ \\
\hline & $5-4$ & $\begin{array}{l}\left(\mathbb{Z}_{4} \times \mathbb{Z}_{2}\right)^{(\mathrm{PG})} \\
\mathbb{Z}_{4} \times \mathbb{Z}_{2}\end{array}$ & $\begin{array}{l}\left(k+\ell+2\left(n_{4}+n_{5}\right), \ell\right) \\
\left(n_{1}+n_{2}+n_{3}, n_{6}\right)\end{array}$ \\
\hline & $5-5$ & $\begin{array}{l}\left(\mathbb{Z}_{4} \times \mathbb{Z}_{4}\right)^{(\mathrm{PG})} \\
\mathbb{Z}_{4}\end{array}$ & $\begin{array}{l}\left(k+2\left(n_{4}+n_{6}\right), \ell+2\left(n_{5}+n_{6}\right)\right) \\
n_{1}+n_{2}+n_{3}\end{array}$ \\
\hline & $6-1$ & $\begin{array}{l}\left(\mathbb{Z}_{2} \times \mathbb{Z}_{2}\right)^{(\mathrm{PG})} \\
\left(\mathbb{Z}_{2}\right)^{4}\end{array}$ & $\begin{array}{l}(k, \ell) \\
\left(n_{1}+n_{2}, n_{3}+n_{4}, n_{5}, n_{6}\right) \\
\end{array}$ \\
\hline & $6-2$ & $\begin{array}{l}\left(\mathbb{Z}_{2} \times \mathbb{Z}_{2}\right)^{(\mathrm{PG})} \\
\left(\mathbb{Z}_{2}\right)^{3}\end{array}$ & $\begin{array}{l}(k, \ell) \\
\left(n_{1}+n_{2}, n_{3}+n_{4}, n_{6}\right)\end{array}$ \\
\hline & $6-3$ & $\begin{array}{l}\left(\mathbb{Z}_{2} \times \mathbb{Z}_{4}\right)^{(\mathrm{PG})} \\
\left(\mathbb{Z}_{2}\right)^{2}\end{array}$ & $\begin{array}{l}\left(k, \ell+2\left(n_{5}+n_{6}\right)\right) \\
\left(n_{1}+n_{2}, n_{3}+n_{4}\right) \\
\end{array}$ \\
\hline & $7-1$ & $\begin{array}{l}\left(\mathbb{Z}_{2} \times \mathbb{Z}_{2}\right)^{(\mathrm{PG})} \\
\left(\mathbb{Z}_{2}\right)^{4}\end{array}$ & $\begin{array}{l}(k, \ell) \\
\left(n_{1}+n_{2}, n_{3}, n_{4}+n_{5}, n_{6}\right)\end{array}$ \\
\hline & $7-2$ & $\begin{array}{l}\left(\mathbb{Z}_{2} \times \mathbb{Z}_{2}\right)^{(\mathrm{PG})} \\
\left(\mathbb{Z}_{2}\right)^{3}\end{array}$ & $\begin{array}{l}(k, \ell) \\
\left(n_{1}+n_{2}, n_{3}, n_{4}+n_{5}\right)\end{array}$ \\
\hline
\end{tabular}

continued... 


\begin{tabular}{|c|c|c|c|}
\hline $\begin{array}{l}\text { Q-class } \\
\text { twist vector }\end{array}$ & $\begin{array}{l}\mathbb{Z} \text { - and } \\
\text { affine class }\end{array}$ & $\begin{array}{l}\text { SG flavor } \\
\text { symmetry } D_{S}\end{array}$ & $\begin{array}{l}\text { discrete } \\
\text { charge }\end{array}$ \\
\hline & $8-1$ & $\begin{array}{l}\left(\mathbb{Z}_{2} \times \mathbb{Z}_{2}\right)^{(\mathrm{PG})} \\
\left(\mathbb{Z}_{2}\right)^{4}\end{array}$ & $\begin{array}{l}(k, \ell) \\
\left(n_{1}, n_{2}+n_{3}, n_{4}, n_{5}+n_{6}\right)\end{array}$ \\
\hline & $9-1$ & $\begin{array}{l}\left(\mathbb{Z}_{2} \times \mathbb{Z}_{2}\right)^{(\mathrm{PG})} \\
\mathbb{Z}_{4} \times\left(\mathbb{Z}_{2}\right)^{2}\end{array}$ & $\begin{array}{l}(k, \ell) \\
\left(n_{1}+n_{2}+n_{3}, n_{4}+n_{5}, n_{6}\right)\end{array}$ \\
\hline & $9-2$ & $\begin{array}{l}\left(\mathbb{Z}_{2} \times \mathbb{Z}_{2}\right)^{(\mathrm{PG})} \\
\mathbb{Z}_{4} \times \mathbb{Z}_{2}\end{array}$ & $\begin{array}{l}(k, \ell) \\
\left(n_{1}+n_{2}+n_{3}, n_{4}+n_{5}\right)\end{array}$ \\
\hline & $9-3$ & $\begin{array}{l}\left(\mathbb{Z}_{2} \times \mathbb{Z}_{2}\right)^{(\mathrm{PG})} \\
\left(\mathbb{Z}_{2}\right)^{3}\end{array}$ & $\begin{array}{l}(k, \ell) \\
\left(n_{1}+n_{2}+n_{3}, n_{4}+n_{5}, n_{6}\right)\end{array}$ \\
\hline & $10-1$ & $\begin{array}{l}\left(\mathbb{Z}_{2} \times \mathbb{Z}_{2}\right)^{(\mathrm{PG})} \\
\mathbb{Z}_{4} \times\left(\mathbb{Z}_{2}\right)^{2}\end{array}$ & $\begin{array}{l}(k, \ell) \\
\left(n_{1}+n_{2}+n_{3}, n_{4}, n_{5}+n_{6}\right)\end{array}$ \\
\hline & $10-2$ & $\begin{array}{l}\left(\mathbb{Z}_{2} \times \mathbb{Z}_{2}\right)^{(\mathrm{PG})} \\
\left(\mathbb{Z}_{2}\right)^{3}\end{array}$ & $\begin{array}{l}(k, \ell) \\
\left(n_{1}+n_{2}+n_{3}, n_{4}, n_{5}+n_{6}\right)\end{array}$ \\
\hline & $11-1$ & $\begin{array}{l}\left(\mathbb{Z}_{2} \times \mathbb{Z}_{2}\right)^{(\mathrm{PG})} \\
\left(\mathbb{Z}_{2}\right)^{3}\end{array}$ & $\begin{array}{l}(k, \ell) \\
\left(n_{1}+n_{2}, n_{3}+n_{4}, n_{5}+n_{6}\right)\end{array}$ \\
\hline & $12-1$ & $\begin{array}{l}\left(\mathbb{Z}_{2} \times \mathbb{Z}_{2}\right)^{(\mathrm{PG})} \\
\left(\mathbb{Z}_{4}\right)^{2}\end{array}$ & $\begin{array}{l}(k, \ell) \\
\left(n_{1}+n_{2}+n_{3}, n_{4}+n_{5}+n_{6}\right)\end{array}$ \\
\hline & $12-2$ & $\begin{array}{l}\left(\mathbb{Z}_{2} \times \mathbb{Z}_{2}\right)^{(\mathrm{PG})} \\
\mathbb{Z}_{4} \times \mathbb{Z}_{2}\end{array}$ & $\begin{array}{l}(k, \ell) \\
\left(n_{1}+n_{2}+n_{3}, n_{4}+n_{5}+n_{6}\right)\end{array}$ \\
\hline \multirow{9}{*}{$\begin{array}{c}\mathbb{Z}_{2} \times \mathbb{Z}_{4} \\
\left(0,0, \frac{1}{2},-\frac{1}{2}\right) \\
\left(0, \frac{1}{4}, 0,-\frac{1}{4}\right)\end{array}$} & $1-1$ & $\begin{array}{l}\left(\mathbb{Z}_{2} \times \mathbb{Z}_{4}\right)^{(\mathrm{PG})} \\
\left(\mathbb{Z}_{2}\right)^{4}\end{array}$ & $\begin{array}{l}(k, \ell) \\
\left(n_{1}+n_{2}, n_{3}, n_{4}, n_{5}+n_{6}\right)\end{array}$ \\
\hline & $1-2$ & $\begin{array}{l}\left(\mathbb{Z}_{2} \times \mathbb{Z}_{4}\right)^{(\mathrm{PG})} \\
\left(\mathbb{Z}_{2}\right)^{3}\end{array}$ & $\begin{array}{l}(k, \ell) \\
\left(n_{3}, n_{4}, n_{5}+n_{6}\right)\end{array}$ \\
\hline & $1-3$ & $\begin{array}{l}\left(\mathbb{Z}_{2} \times \mathbb{Z}_{4}\right)^{(\mathrm{PG})} \\
\left(\mathbb{Z}_{2}\right)^{3}\end{array}$ & $\begin{array}{l}(k, \ell) \\
\left(n_{1}+n_{2}+n_{5}+n_{6}, n_{3}, n_{4}\right)\end{array}$ \\
\hline & $1-4$ & $\begin{array}{l}\left(\mathbb{Z}_{2} \times \mathbb{Z}_{4}\right)^{(\mathrm{PG})} \\
\left(\mathbb{Z}_{2}\right)^{3}\end{array}$ & $\begin{array}{l}(k, \ell) \\
\left(n_{1}+n_{2}, n_{3}, n_{5}+n_{6}\right)\end{array}$ \\
\hline & $1-5$ & $\begin{array}{l}\left(\mathbb{Z}_{2} \times \mathbb{Z}_{4}\right)^{(\mathrm{PG})} \\
\left(\mathbb{Z}_{2}\right)^{3}\end{array}$ & $\begin{array}{l}(k, \ell) \\
\left(n_{1}+n_{2}+n_{4}, n_{3}, n_{5}+n_{6}\right)\end{array}$ \\
\hline & $1-6$ & $\begin{array}{l}\left(\mathbb{Z}_{2} \times \mathbb{Z}_{4}\right)^{(\mathrm{PG})} \\
\left(\mathbb{Z}_{2}\right)^{3}\end{array}$ & $\begin{array}{l}(k, \ell) \\
\left(n_{1}+n_{2}+n_{4}, n_{3}, n_{4}+n_{5}+n_{6}\right)\end{array}$ \\
\hline & $2-1$ & $\begin{array}{l}\left(\mathbb{Z}_{2} \times \mathbb{Z}_{4}\right)^{(\mathrm{PG})} \\
\left(\mathbb{Z}_{2}\right)^{4}\end{array}$ & $\begin{array}{l}(k, \ell) \\
\left(n_{1}+n_{2}, n_{3}+n_{4}, n_{5}, n_{6}\right)\end{array}$ \\
\hline & $2-2$ & $\begin{array}{l}\left(\mathbb{Z}_{2} \times \mathbb{Z}_{4}\right)^{(\mathrm{PG})} \\
\left(\mathbb{Z}_{2}\right)^{3}\end{array}$ & $\begin{array}{l}(k, \ell) \\
\left(n_{1}+n_{2}, n_{3}+n_{4}, n_{5}\right) \\
\end{array}$ \\
\hline & $2-3$ & $\begin{array}{l}\left(\mathbb{Z}_{4} \times \mathbb{Z}_{4}\right)^{(\mathrm{PG})} \\
\left(\mathbb{Z}_{2}\right)^{2}\end{array}$ & $\begin{array}{l}\left(k+2\left(n_{1}+n_{2}+n_{3}+n_{4}\right), \ell\right) \\
\left(n_{5}, n_{6}\right)\end{array}$ \\
\hline
\end{tabular}

continued... 


\begin{tabular}{|c|c|c|c|}
\hline $\begin{array}{c}\text { Q-class } \\
\text { twist vector }\end{array}$ & $\begin{array}{c}\mathbb{Z} \text { - and } \\
\text { affine class }\end{array}$ & $\begin{array}{l}\text { SG flavor } \\
\text { symmetry } D_{S}\end{array}$ & $\begin{array}{l}\text { discrete } \\
\text { charge }\end{array}$ \\
\hline & $2-4$ & $\begin{array}{l}\left(\mathbb{Z}_{4} \times \mathbb{Z}_{4}\right)^{(\mathrm{PG})} \\
\left(\mathbb{Z}_{2}\right)^{2}\end{array}$ & $\begin{array}{l}\left(k+2\left(n_{3}+n_{4}+n_{6}\right), \ell\right) \\
\left(n_{1}+n_{2}+n_{6}, n_{5}\right)\end{array}$ \\
\hline & $2-5$ & $\begin{array}{l}\left(\mathbb{Z}_{2} \times \mathbb{Z}_{4}\right)^{(\mathrm{PG})} \\
\left(\mathbb{Z}_{2}\right)^{3}\end{array}$ & $\begin{array}{l}(k, \ell) \\
\left(n_{1}+n_{2}, n_{5}, n_{6}\right)\end{array}$ \\
\hline & $2-6$ & $\begin{array}{l}\left(\mathbb{Z}_{2} \times \mathbb{Z}_{4}\right)^{(\mathrm{PG})} \\
\left(\mathbb{Z}_{2}\right)^{3}\end{array}$ & $\begin{array}{l}(k, \ell) \\
\left(n_{1}+n_{2}, n_{3}+n_{4}+n_{6}, n_{5}\right)\end{array}$ \\
\hline & $3-1$ & $\begin{array}{l}\left(\mathbb{Z}_{2} \times \mathbb{Z}_{4}\right)^{(\mathrm{PG})} \\
\left(\mathbb{Z}_{2}\right)^{3}\end{array}$ & $\begin{array}{l}(k, \ell) \\
\left(n_{1}+n_{2}+n_{3}, n_{4}, n_{5}+n_{6}\right)\end{array}$ \\
\hline & $3-2$ & $\begin{array}{l}\left(\mathbb{Z}_{2} \times \mathbb{Z}_{4}\right)^{(\mathrm{PG})} \\
\left(\mathbb{Z}_{2}\right)^{2}\end{array}$ & $\begin{array}{l}(k, \ell) \\
\left(n_{1}+n_{2}+n_{3}, n_{4}\right)\end{array}$ \\
\hline & $3-3$ & $\begin{array}{l}\left(\mathbb{Z}_{2} \times \mathbb{Z}_{4}\right)^{(\mathrm{PG})} \\
\left(\mathbb{Z}_{2}\right)^{2}\end{array}$ & $\begin{array}{l}(k, \ell) \\
\left(n_{1}+n_{2}+n_{3}, n_{5}+n_{6}\right)\end{array}$ \\
\hline & $3-4$ & $\begin{array}{l}\left(\mathbb{Z}_{2} \times \mathbb{Z}_{4}\right)^{(\mathrm{PG})} \\
\left(\mathbb{Z}_{2}\right)^{2}\end{array}$ & $\begin{array}{l}(k, \ell) \\
\left(n_{1}+n_{2}+n_{3}, n_{4}+n_{5}+n_{6}\right)\end{array}$ \\
\hline & $3-5$ & $\begin{array}{l}\left(\mathbb{Z}_{2} \times \mathbb{Z}_{4}\right)^{(\mathrm{PG})} \\
\left(\mathbb{Z}_{2}\right)^{2}\end{array}$ & $\begin{array}{l}(k, \ell) \\
\left(n_{4}, n_{5}+n_{6}\right)\end{array}$ \\
\hline & $3-6$ & $\begin{array}{l}\left(\mathbb{Z}_{2} \times \mathbb{Z}_{4}\right)^{(\mathrm{PG})} \\
\left(\mathbb{Z}_{2}\right)^{2}\end{array}$ & $\begin{array}{l}(k, \ell) \\
\left(n_{1}+n_{2}+n_{3}+n_{5}+n_{6}, n_{4}\right)\end{array}$ \\
\hline & $4-1$ & $\begin{array}{l}\left(\mathbb{Z}_{2} \times \mathbb{Z}_{4}\right)^{(\mathrm{PG})} \\
\left(\mathbb{Z}_{2}\right)^{3}\end{array}$ & $\begin{array}{l}(k, \ell) \\
\left(n_{1}+n_{2}, n_{3}+n_{4}+n_{5}, n_{6}\right)\end{array}$ \\
\hline & $4-2$ & $\begin{array}{l}\left(\mathbb{Z}_{2} \times \mathbb{Z}_{4}\right)^{(\mathrm{PG})} \\
\left(\mathbb{Z}_{2}\right)^{2}\end{array}$ & $\begin{array}{l}(k, \ell) \\
\left(n_{1}+n_{2}, n_{3}+n_{4}+n_{5}\right)\end{array}$ \\
\hline & $4-3$ & $\begin{array}{l}\left(\mathbb{Z}_{2} \times \mathbb{Z}_{4}\right)^{(\mathrm{PG})} \\
\left(\mathbb{Z}_{2}\right)^{2}\end{array}$ & $\begin{array}{l}(k, \ell) \\
\left(n_{1}+n_{2}+n_{3}+n_{4}+n_{5}, n_{6}\right)\end{array}$ \\
\hline & $4-4$ & $\begin{array}{l}\left(\mathbb{Z}_{2} \times \mathbb{Z}_{4}\right)^{(\mathrm{PG})} \\
\left(\mathbb{Z}_{2}\right)^{2}\end{array}$ & $\begin{array}{l}(k, \ell) \\
\left(n_{1}+n_{2}+n_{6}, n_{3}+n_{4}+n_{5}+n_{6}\right)\end{array}$ \\
\hline & $4-5$ & $\begin{array}{l}\left(\mathbb{Z}_{2} \times \mathbb{Z}_{4}\right)^{(\mathrm{PG})} \\
\left(\mathbb{Z}_{2}\right)^{2}\end{array}$ & $\begin{array}{l}(k, \ell) \\
\left(n_{1}+n_{2}, n_{6}\right)\end{array}$ \\
\hline & $5-1$ & $\begin{array}{l}\left(\mathbb{Z}_{2} \times \mathbb{Z}_{4}\right)^{(\mathrm{PG})} \\
\left(\mathbb{Z}_{2}\right)^{3}\end{array}$ & $\begin{array}{l}(k, \ell) \\
\left(n_{1}+n_{2}+n_{3}+n_{4}, n_{5}, n_{6}\right)\end{array}$ \\
\hline & $5-2$ & $\begin{array}{l}\left(\mathbb{Z}_{2} \times \mathbb{Z}_{4}\right)^{(\mathrm{PG})} \\
\left(\mathbb{Z}_{2}\right)^{2}\end{array}$ & $\begin{array}{l}(k, \ell) \\
\left(n_{1}+n_{2}+n_{3}+n_{4}, n_{5}\right) \\
\end{array}$ \\
\hline & $6-1$ & $\begin{array}{l}\left(\mathbb{Z}_{2} \times \mathbb{Z}_{4}\right)^{(\mathrm{PG})} \\
\left(\mathbb{Z}_{2}\right)^{3}\end{array}$ & $\begin{array}{l}(k, \ell) \\
\left(n_{1}+n_{2}+n_{4}+n_{5}, n_{3}, n_{6}\right)\end{array}$ \\
\hline & $6-2$ & $\begin{array}{l}\left(\mathbb{Z}_{2} \times \mathbb{Z}_{4}\right)^{(\mathrm{PG})} \\
\left(\mathbb{Z}_{2}\right)^{2}\end{array}$ & $\begin{array}{l}(k, \ell) \\
\left(n_{3}, n_{6}\right)\end{array}$ \\
\hline
\end{tabular}

continued... 


\begin{tabular}{|c|c|c|c|}
\hline $\begin{array}{l}\text { Q-class } \\
\text { twist vector }\end{array}$ & $\begin{array}{l}\mathbb{Z} \text { - and } \\
\text { affine class }\end{array}$ & $\begin{array}{l}\text { SG flavor } \\
\text { symmetry } D_{S}\end{array}$ & $\begin{array}{l}\text { discrete } \\
\text { charge }\end{array}$ \\
\hline & 6-3 & $\begin{array}{l}\left(\mathbb{Z}_{2} \times \mathbb{Z}_{4}\right)^{(\mathrm{PG})} \\
\left(\mathbb{Z}_{2}\right)^{2}\end{array}$ & $\begin{array}{l}(k, \ell) \\
\left(n_{1}+n_{2}+n_{4}+n_{5}, n_{3}\right)\end{array}$ \\
\hline & $6-4$ & $\begin{array}{l}\left(\mathbb{Z}_{2} \times \mathbb{Z}_{4}\right)^{(\mathrm{PG})} \\
\left(\mathbb{Z}_{2}\right)^{2}\end{array}$ & $\begin{array}{l}(k, \ell) \\
\left(n_{1}+n_{2}+n_{4}+n_{5}+n_{6}, n_{3}\right)\end{array}$ \\
\hline & $6-5$ & $\begin{array}{l}\left(\mathbb{Z}_{4} \times \mathbb{Z}_{4}\right)^{(\mathrm{PG})} \\
\mathbb{Z}_{2}\end{array}$ & $\begin{array}{l}\left(k+2\left(n_{1}+n_{2}+n_{3}+n_{4}+n_{5}\right), \ell\right) \\
n_{6}\end{array}$ \\
\hline & $7-1$ & $\begin{array}{l}\left(\mathbb{Z}_{2} \times \mathbb{Z}_{4}\right)^{(\mathrm{PG})} \\
\left(\mathbb{Z}_{2}\right)^{3}\end{array}$ & $\begin{array}{l}(k, \ell) \\
\left(n_{1}+n_{2}+n_{3}+n_{4}, n_{5}, n_{6}\right)\end{array}$ \\
\hline & $7-2$ & $\begin{array}{l}\left(\mathbb{Z}_{2} \times \mathbb{Z}_{4}\right)^{(\mathrm{PG})} \\
\left(\mathbb{Z}_{2}\right)^{2}\end{array}$ & $\begin{array}{l}(k, \ell) \\
\left(n_{1}+n_{2}+n_{3}+n_{4}, n_{5}\right)\end{array}$ \\
\hline & $7-3$ & $\begin{array}{l}\left(\mathbb{Z}_{2} \times \mathbb{Z}_{4}\right)^{(\mathrm{PG})} \\
\left(\mathbb{Z}_{2}\right)^{2}\end{array}$ & $\begin{array}{l}(k, \ell) \\
\left(n_{1}+n_{2}+n_{3}+n_{4}, n_{6}\right)\end{array}$ \\
\hline & $8-1$ & $\begin{array}{l}\left(\mathbb{Z}_{2} \times \mathbb{Z}_{4}\right)^{(\mathrm{PG})} \\
\left(\mathbb{Z}_{2}\right)^{2}\end{array}$ & $\begin{array}{l}(k, \ell) \\
\left(n_{1}+n_{2}+n_{3}, n_{4}+n_{5}+n_{6}\right)\end{array}$ \\
\hline & $8-2$ & $\begin{array}{l}\left(\mathbb{Z}_{2} \times \mathbb{Z}_{4}\right)^{(\mathrm{PG})} \\
\mathbb{Z}_{2}\end{array}$ & $\begin{array}{l}(k, \ell) \\
n_{4}+n_{5}+n_{6}\end{array}$ \\
\hline & $8-3$ & $\begin{array}{l}\left(\mathbb{Z}_{2} \times \mathbb{Z}_{4}\right)^{(\mathrm{PG})} \\
\mathbb{Z}_{2}\end{array}$ & $\begin{array}{l}(k, \ell) \\
n_{1}+n_{2}+n_{3}+n_{4}+n_{5}+n_{6}\end{array}$ \\
\hline & $9-1$ & $\begin{array}{l}\left(\mathbb{Z}_{2} \times \mathbb{Z}_{4}\right)^{(\mathrm{PG})} \\
\mathbb{Z}_{4} \times \mathbb{Z}_{2}\end{array}$ & $\begin{array}{l}(k, \ell) \\
\left(n_{1}+n_{2}+n_{3}+n_{4}+n_{5}, n_{6}\right)\end{array}$ \\
\hline & $9-2$ & $\begin{array}{l}\left(\mathbb{Z}_{2} \times \mathbb{Z}_{4}\right)^{(\mathrm{PG})} \\
\mathbb{Z}_{4}\end{array}$ & $\begin{array}{l}(k, \ell) \\
n_{1}+n_{2}+n_{3}+n_{4}+n_{5}\end{array}$ \\
\hline & $9-3$ & $\begin{array}{l}\left(\mathbb{Z}_{2} \times \mathbb{Z}_{4}\right)^{(\mathrm{PG})} \\
\left(\mathbb{Z}_{2}\right)^{2}\end{array}$ & $\begin{array}{l}(k, \ell) \\
\left(n_{1}+n_{2}+n_{3}+n_{4}+n_{5}, n_{6}\right)\end{array}$ \\
\hline & $10-1$ & $\begin{array}{l}\left(\mathbb{Z}_{2} \times \mathbb{Z}_{4}\right)^{(\mathrm{PG})} \\
\left(\mathbb{Z}_{2}\right)^{2}\end{array}$ & $\begin{array}{l}(k, \ell) \\
\left(n_{1}+n_{2}+n_{3}+n_{4}+n_{5}, n_{6}\right)\end{array}$ \\
\hline & $10-2$ & $\begin{array}{l}\left(\mathbb{Z}_{2} \times \mathbb{Z}_{4}\right)^{(\mathrm{PG})} \\
\mathbb{Z}_{2}\end{array}$ & $\begin{array}{l}(k, \ell) \\
n_{1}+n_{2}+n_{3}+n_{4}+n_{5}\end{array}$ \\
\hline \multirow{4}{*}{$\begin{array}{c}\mathbb{Z}_{2} \times \mathbb{Z}_{6}-\mathrm{I} \\
\left(0,0, \frac{1}{2},-\frac{1}{2}\right) \\
\left(0, \frac{1}{6}, 0,-\frac{1}{6}\right)\end{array}$} & $1-1$ & $\begin{array}{l}\left(\mathbb{Z}_{2} \times \mathbb{Z}_{6}\right)^{(\mathrm{PG})} \\
\left(\mathbb{Z}_{2}\right)^{2}\end{array}$ & $\begin{array}{l}(k, \ell) \\
\left(n_{3}, n_{4}\right)\end{array}$ \\
\hline & $1-2$ & $\begin{array}{l}\left(\mathbb{Z}_{2} \times \mathbb{Z}_{6}\right)^{(\mathrm{PG})} \\
\mathbb{Z}_{2}\end{array}$ & $\begin{array}{l}(k, \ell) \\
n_{3}\end{array}$ \\
\hline & $2-1$ & $\begin{array}{l}\left(\mathbb{Z}_{2} \times \mathbb{Z}_{6}\right)^{(\mathrm{PG})} \\
\left(\mathbb{Z}_{2}\right)^{2}\end{array}$ & $\begin{array}{l}(k, \ell) \\
\left(n_{5}, n_{6}\right)\end{array}$ \\
\hline & $2-2$ & $\begin{array}{l}\left(\mathbb{Z}_{2} \times \mathbb{Z}_{6}\right)^{(\mathrm{PG})} \\
\mathbb{Z}_{2}\end{array}$ & $\begin{array}{l}(k, \ell) \\
n_{5} \\
\end{array}$ \\
\hline
\end{tabular}

continued... 


\begin{tabular}{|c|c|c|c|}
\hline $\begin{array}{l}\text { Q-class } \\
\text { twist vector }\end{array}$ & $\begin{array}{c}\mathbb{Z} \text { - and } \\
\text { affine class }\end{array}$ & $\begin{array}{l}\text { SG flavor } \\
\text { symmetry } D_{S}\end{array}$ & $\begin{array}{l}\text { discrete } \\
\text { charge }\end{array}$ \\
\hline \multirow{4}{*}{$\begin{array}{l}\mathbb{Z}_{2} \times \mathbb{Z}_{6}-\mathrm{II} \\
\left(0,0, \frac{1}{2},-\frac{1}{2}\right) \\
\left(0, \frac{1}{6}, \frac{1}{6},-\frac{1}{3}\right)\end{array}$} & $1-1$ & $\left(\mathbb{Z}_{2} \times \mathbb{Z}_{6}\right)^{(\mathrm{PG})}$ & $(k, \ell)$ \\
\hline & $2-1$ & $\left(\mathbb{Z}_{2} \times \mathbb{Z}_{6}\right)^{(\mathrm{PG})}$ & $(k, \ell)$ \\
\hline & $3-1$ & $\left(\mathbb{Z}_{2} \times \mathbb{Z}_{6}\right)^{(\mathrm{PG})}$ & $(k, \ell)$ \\
\hline & $4-1$ & $\left(\mathbb{Z}_{2} \times \mathbb{Z}_{6}\right)^{(\mathrm{PG})}$ & $(k, \ell)$ \\
\hline \multirow{15}{*}{$\begin{array}{c}\mathbb{Z}_{3} \times \mathbb{Z}_{3} \\
\left(0,0, \frac{1}{3},-\frac{1}{3}\right) \\
\left(0, \frac{1}{3}, 0,-\frac{1}{3}\right)\end{array}$} & $1-1$ & $\begin{array}{l}\left(\mathbb{Z}_{3} \times \mathbb{Z}_{3}\right)^{(\mathrm{PG})} \\
\left(\mathbb{Z}_{3}\right)^{3}\end{array}$ & $\begin{array}{l}(k, \ell) \\
\left(n_{1}+n_{2}, n_{3}+n_{4}, n_{5}+n_{6}\right)\end{array}$ \\
\hline & $1-2$ & $\begin{array}{l}\left(\mathbb{Z}_{3} \times \mathbb{Z}_{3}\right)^{(\mathrm{PG})} \\
\left(\mathbb{Z}_{3}\right)^{2}\end{array}$ & $\begin{array}{l}(k, \ell) \\
\left(n_{1}+n_{2}, n_{3}+n_{4}\right)\end{array}$ \\
\hline & $1-3$ & $\begin{array}{l}\left(\mathbb{Z}_{3} \times \mathbb{Z}_{3}\right)^{(\mathrm{PG})} \\
\left(\mathbb{Z}_{3}\right)^{2}\end{array}$ & $\begin{array}{l}(k, \ell) \\
\left(2\left(n_{1}+n_{2}\right)+n_{5}+n_{6}, n_{3}+n_{4}\right)\end{array}$ \\
\hline & $1-4$ & $\begin{array}{l}\left(\mathbb{Z}_{3} \times \mathbb{Z}_{3}\right)^{(\mathrm{PG})} \\
\left(\mathbb{Z}_{3}\right)^{2}\end{array}$ & $\begin{array}{l}(k, \ell) \\
\left(n_{1}+n_{2}+2\left(n_{3}+n_{4}\right), 2\left(n_{3}+n_{4}\right)+n_{5}+n_{6}\right)\end{array}$ \\
\hline & $2-1$ & $\begin{array}{l}\left(\mathbb{Z}_{3} \times \mathbb{Z}_{3}\right)^{(\mathrm{PG})} \\
\left(\mathbb{Z}_{3}\right)^{2}\end{array}$ & $\begin{array}{l}(k, \ell) \\
\left(n_{1}+n_{2}+n_{3}+n_{4}, n_{5}+n_{6}\right)\end{array}$ \\
\hline & $2-2$ & $\begin{array}{l}\left(\mathbb{Z}_{3} \times \mathbb{Z}_{3}\right)^{(\mathrm{PG})} \\
\mathbb{Z}_{3}\end{array}$ & $\begin{array}{l}(k, \ell) \\
n_{1}+n_{2}+n_{3}+n_{4}\end{array}$ \\
\hline & $2-3$ & $\begin{array}{l}\left(\mathbb{Z}_{3} \times \mathbb{Z}_{3}\right)^{(\mathrm{PG})} \\
\mathbb{Z}_{3}\end{array}$ & $\begin{array}{l}(k, \ell) \\
n_{5}+n_{6}\end{array}$ \\
\hline & $2-4$ & $\begin{array}{l}\left(\mathbb{Z}_{3} \times \mathbb{Z}_{3}\right)^{(\mathrm{PG})} \\
\mathbb{Z}_{3}\end{array}$ & $\begin{array}{l}(k, \ell) \\
n_{1}+n_{2}+n_{3}+n_{4}+2\left(n_{5}+n_{6}\right)\end{array}$ \\
\hline & $3-1$ & $\begin{array}{l}\left(\mathbb{Z}_{3} \times \mathbb{Z}_{3}\right)^{(\mathrm{PG})} \\
\left(\mathbb{Z}_{3}\right)^{2}\end{array}$ & $\begin{array}{l}(k, \ell) \\
\left(n_{1}+n_{2}+n_{3}+n_{4}, n_{5}+n_{6}\right)\end{array}$ \\
\hline & $3-2$ & $\begin{array}{l}\left(\mathbb{Z}_{3} \times \mathbb{Z}_{3}\right)^{(\mathrm{PG})} \\
\mathbb{Z}_{3}\end{array}$ & $\begin{array}{l}(k, \ell) \\
n_{5}+n_{6}\end{array}$ \\
\hline & $3-3$ & $\begin{array}{l}\left(\mathbb{Z}_{3} \times \mathbb{Z}_{3}\right)^{(\mathrm{PG})} \\
\mathbb{Z}_{3}\end{array}$ & $\begin{array}{l}(k, \ell) \\
n_{1}+n_{2}+n_{3}+n_{4}+n_{5}+n_{6}\end{array}$ \\
\hline & $4-1$ & $\begin{array}{l}\left(\mathbb{Z}_{3} \times \mathbb{Z}_{3}\right)^{(\mathrm{PG})} \\
\left(\mathbb{Z}_{3}\right)^{2}\end{array}$ & $\begin{array}{l}(k, \ell) \\
\left(n_{1}+n_{4}, n_{2}+n_{3}+n_{5}+n_{6}\right)\end{array}$ \\
\hline & $4-2$ & $\begin{array}{l}\left(\mathbb{Z}_{3} \times \mathbb{Z}_{3}\right)^{(\mathrm{PG})} \\
\mathbb{Z}_{3}\end{array}$ & $\begin{array}{l}(k, \ell) \\
n_{1}+n_{4}\end{array}$ \\
\hline & $4-3$ & $\left(\mathbb{Z}_{9} \times \mathbb{Z}_{3}\right)^{(\mathrm{PG})}$ & $\left(k+2 \ell+6\left(n_{1}+n_{2}+n_{3}+n_{4}+n_{5}+n_{6}\right), \ell\right)$ \\
\hline & $5-1$ & $\begin{array}{l}\left(\mathbb{Z}_{3} \times \mathbb{Z}_{3}\right)^{(\mathrm{PG})} \\
\mathbb{Z}_{3}\end{array}$ & $\begin{array}{l}(k, \ell) \\
n_{1}+n_{2}+n_{3}+n_{4}+n_{5}+n_{6}\end{array}$ \\
\hline \multirow{2}{*}{$\begin{array}{c}\mathbb{Z}_{3} \times \mathbb{Z}_{6} \\
\left(0,0, \frac{1}{3},-\frac{1}{3}\right) \\
\left(0, \frac{1}{6}, 0,-\frac{1}{6}\right)\end{array}$} & $1-1$ & $\begin{array}{l}\left(\mathbb{Z}_{3} \times \mathbb{Z}_{6}\right)^{(\mathrm{PG})} \\
\mathbb{Z}_{3}\end{array}$ & $\begin{array}{l}(k, \ell) \\
n_{3}+n_{4}\end{array}$ \\
\hline & $1-2$ & $\left(\mathbb{Z}_{3} \times \mathbb{Z}_{6}\right)^{(\mathrm{PG})}$ & $(k, \ell)$ \\
\hline
\end{tabular}

continued... 


\begin{tabular}{|c|c|c|c|}
\hline $\begin{array}{c}\text { Q-class } \\
\text { twist vector }\end{array}$ & $\begin{array}{c}\mathbb{Z} \text { - and } \\
\text { affine class }\end{array}$ & $\begin{array}{l}\text { SG flavor } \\
\text { symmetry } D_{S}\end{array}$ & $\begin{array}{l}\text { discrete } \\
\text { charge }\end{array}$ \\
\hline & $2-1$ & $\begin{array}{l}\left(\mathbb{Z}_{3} \times \mathbb{Z}_{6}\right)^{(\mathrm{PG})} \\
\mathbb{Z}_{3}\end{array}$ & $\begin{array}{l}(k, \ell) \\
n_{5}+n_{6}\end{array}$ \\
\hline & $2-2$ & $\left(\mathbb{Z}_{3} \times \mathbb{Z}_{6}\right)^{(\mathrm{PG})}$ & $(k, \ell)$ \\
\hline \multirow{15}{*}{$\begin{array}{c}\mathbb{Z}_{4} \times \mathbb{Z}_{4} \\
\left(0,0, \frac{1}{4},-\frac{1}{4}\right) \\
\left(0, \frac{1}{4}, 0,-\frac{1}{4}\right)\end{array}$} & $1-1$ & $\begin{array}{l}\left(\mathbb{Z}_{4} \times \mathbb{Z}_{4}\right)^{(\mathrm{PG})} \\
\left(\mathbb{Z}_{2}\right)^{3}\end{array}$ & $\begin{array}{l}(k, \ell) \\
\left(n_{1}+n_{2}, n_{3}+n_{4}, n_{5}+n_{6}\right)\end{array}$ \\
\hline & $1-2$ & $\begin{array}{l}\left(\mathbb{Z}_{4} \times \mathbb{Z}_{4}\right)^{(\mathrm{PG})} \\
\left(\mathbb{Z}_{2}\right)^{2}\end{array}$ & $\begin{array}{l}(k, \ell) \\
\left(n_{1}+n_{2}, n_{3}+n_{4}\right)\end{array}$ \\
\hline & $1-3$ & $\begin{array}{l}\left(\mathbb{Z}_{4} \times \mathbb{Z}_{4}\right)^{(\mathrm{PG})} \\
\left(\mathbb{Z}_{2}\right)^{2}\end{array}$ & $\begin{array}{l}(k, \ell) \\
\left(n_{1}+n_{2}+n_{5}+n_{6}, n_{3}+n_{4}\right)\end{array}$ \\
\hline & $1-4$ & $\begin{array}{l}\left(\mathbb{Z}_{4} \times \mathbb{Z}_{4}\right)^{(\mathrm{PG})} \\
\left(\mathbb{Z}_{2}\right)^{2}\end{array}$ & $\begin{array}{l}(k, \ell) \\
\left(n_{1}+n_{2}+n_{3}+n_{4}, n_{3}+n_{4}+n_{5}+n_{6}\right)\end{array}$ \\
\hline & $2-1$ & $\begin{array}{l}\left(\mathbb{Z}_{4} \times \mathbb{Z}_{4}\right)^{(\mathrm{PG})} \\
\left(\mathbb{Z}_{2}\right)^{2}\end{array}$ & $\begin{array}{l}(k, \ell) \\
\left(n_{1}+n_{2}+n_{3}+n_{4}, n_{5}+n_{6}\right)\end{array}$ \\
\hline & $2-2$ & $\begin{array}{l}\left(\mathbb{Z}_{4} \times \mathbb{Z}_{4}\right)^{(\mathrm{PG})} \\
\mathbb{Z}_{2}\end{array}$ & $\begin{array}{l}(k, \ell) \\
n_{5}+n_{6}\end{array}$ \\
\hline & $2-3$ & $\begin{array}{l}\left(\mathbb{Z}_{4} \times \mathbb{Z}_{4}\right)^{(\mathrm{PG})} \\
\mathbb{Z}_{2}\end{array}$ & $\begin{array}{l}(k, \ell) \\
n_{1}+n_{2}+n_{3}+n_{4}\end{array}$ \\
\hline & $2-4$ & $\begin{array}{l}\left(\mathbb{Z}_{4} \times \mathbb{Z}_{4}\right)^{(\mathrm{PG})} \\
\mathbb{Z}_{2}\end{array}$ & $\begin{array}{l}(k, \ell) \\
n_{1}+n_{2}+n_{3}+n_{4}+n_{5}+n_{6}\end{array}$ \\
\hline & $3-1$ & $\begin{array}{l}\left(\mathbb{Z}_{4} \times \mathbb{Z}_{4}\right)^{(\mathrm{PG})} \\
\left(\mathbb{Z}_{2}\right)^{2}\end{array}$ & $\begin{array}{l}(k, \ell) \\
\left(n_{1}+n_{2}, n_{3}+n_{4}+n_{5}+n_{6}\right)\end{array}$ \\
\hline & $3-2$ & $\begin{array}{l}\left(\mathbb{Z}_{4} \times \mathbb{Z}_{4}\right)^{(\mathrm{PG})} \\
\mathbb{Z}_{2}\end{array}$ & $\begin{array}{l}(k, \ell) \\
n_{1}+n_{2}+n_{3}+n_{4}+n_{5}+n_{6}\end{array}$ \\
\hline & $4-1$ & $\begin{array}{l}\left(\mathbb{Z}_{4} \times \mathbb{Z}_{4}\right)^{(\mathrm{PG})} \\
\left(\mathbb{Z}_{2}\right)^{2}\end{array}$ & $\begin{array}{l}(k, \ell) \\
\left(n_{1}+n_{2}, n_{3}+n_{4}+n_{5}+n_{6}\right)\end{array}$ \\
\hline & $4-2$ & $\begin{array}{l}\left(\mathbb{Z}_{4} \times \mathbb{Z}_{4}\right)^{(\mathrm{PG})} \\
\mathbb{Z}_{2}\end{array}$ & $\begin{array}{l}(k, \ell) \\
n_{3}+n_{4}+n_{5}+n_{6}\end{array}$ \\
\hline & $4-3$ & $\begin{array}{l}\left(\mathbb{Z}_{4} \times \mathbb{Z}_{4}\right)^{(\mathrm{PG})} \\
\mathbb{Z}_{2}\end{array}$ & $\begin{array}{l}(k, \ell) \\
n_{1}+n_{2}\end{array}$ \\
\hline & $5-1$ & $\begin{array}{l}\left(\mathbb{Z}_{4} \times \mathbb{Z}_{4}\right)^{(\mathrm{PG})} \\
\mathbb{Z}_{2}\end{array}$ & $\begin{array}{l}(k, \ell) \\
n_{1}+n_{2}+n_{3}+n_{4}+n_{5}+n_{6}\end{array}$ \\
\hline & $5-2$ & $\left(\mathbb{Z}_{4} \times \mathbb{Z}_{4}\right)^{(\mathrm{PG})}$ & $(k, \ell)$ \\
\hline $\begin{array}{c}\mathbb{Z}_{6} \times \mathbb{Z}_{6} \\
\left(0,0, \frac{1}{6},-\frac{1}{6}\right) \\
\left(0, \frac{1}{6}, 0,-\frac{1}{6}\right)\end{array}$ & $1-1$ & $\left(\mathbb{Z}_{6} \times \mathbb{Z}_{6}\right)^{(\mathrm{PG})}$ & $(k, \ell)$ \\
\hline
\end{tabular}

Table 1. Space group flavor symmetries obtained from the space group selection rule for all space groups with Abelian point group and $\mathcal{N}=1$ supersymmetry [25, 26]. 


\subsection{Discrete anomalies of the SG flavor symmetry $D_{S}$}

Discrete groups can be anomalous and their anomaly coefficients can be computed directly using Fujikawa's method for the Jacobian of the path integral measure [37, 38]. Following refs. $[27,39]$, the mixed $\mathbb{Z}_{N}-G_{i}-G_{i}$ anomaly coefficient of a discrete group $\mathbb{Z}_{N}$ and a non-Abelian gauge group factor $G_{i}$ is given by

$$
A_{\mathbb{Z}_{N}-G_{i}-G_{i}}=\sum_{\boldsymbol{r}^{(f)}} q^{(f)} \ell\left(\boldsymbol{r}^{(f)}\right) .
$$

Here, the summation runs over all fermions that transform in the representation $\boldsymbol{r}^{(f)}$ of the gauge group factor $G_{i}$ and $q^{(f)}$ denotes their discrete $\mathbb{Z}_{N}$ charge as given in table 1 for a string with constructing element ${ }^{3}$

$$
g=\left(T_{1}\right)^{n_{1}} \ldots\left(T_{6}\right)^{n_{6}}\left(g_{\theta}\right)^{k}\left(g_{\omega}\right)^{\ell} \in S,
$$

with $n_{1}, \ldots, n_{6}, k, \ell \in \mathbb{Z}$. In addition, the Dynkin index $\ell\left(\boldsymbol{r}^{(f)}\right)$ is normalized such that $\ell(\boldsymbol{N})=1$ for the fundamental representation $\boldsymbol{N}$ of $\mathrm{SU}(N)$ and $\ell(\mathbf{2} \boldsymbol{N})=2$ for the vector representation $\mathbf{2} \boldsymbol{N}$ of $\mathrm{SO}(2 N)$. Furthermore, the $\mathbb{Z}_{N}$ - grav. - grav. anomaly coefficient reads

$$
A_{\mathbb{Z}_{N} \text {-grav.-grav. }}=\sum_{m} q^{(m)} \operatorname{dim}\left(\boldsymbol{R}^{(m)}\right),
$$

where the summation runs over all fermions that transform in the representation $\boldsymbol{R}^{(m)}$ of $G_{1} \times G_{2} \times \ldots$, being the full non-Abelian gauge group of the theory.

For all space groups from table 1 we have constructed more than 1,000 random orbifold models using the orbifolder [36]. For every orbifold model we have checked that for each $\mathbb{Z}_{N}$ factor of $D_{S}$ there is a discrete Green-Schwarz constant $\Delta_{\text {GS }}[27,40]$ such that for all non-Abelian gauge group factors $G_{i}$ the anomalies are universal, i.e.

$$
\begin{aligned}
A_{\mathbb{Z}_{N}-G_{i}-G_{i}} & =\Delta_{\mathrm{GS}} \bmod N, \\
A_{\mathbb{Z}_{N} \text {-grav.-grav. }} & =12 A_{\mathbb{Z}_{N}-G_{i}-G_{i}} \bmod N .
\end{aligned}
$$

Consequently, if $\Delta_{\mathrm{GS}} \neq 0$ the universal discrete anomalies can be canceled by a discrete Green-Schwarz mechanism involving a single, universally coupled axion. Thus, we have performed a non-trivial test of all SG flavor symmetries.

\section{Conclusions}

There are large sets of semi-realistic string orbifold models, whose phenomenology may reveal interesting features of the string landscape, restricting thereby string constructions in the search of an ultraviolet completion of low-energy physics. Studying the phenomenology of orbifold compactifications requires, as one of the first steps, the identification and understanding of all symmetries of a string model.

\footnotetext{
${ }^{3}$ Note that in the presence of roto-translations this constructing element $g$ is equal to $g=\left(\theta^{k} \omega^{\ell}, \lambda_{(k, \ell)}+\right.$ $\left.n_{i} e_{i}\right)$ for some non-trivial translation $\lambda_{(k, \ell)}$. The discrete charges $q^{(f)}$, however, do not depend on $\lambda_{(k, \ell)}$ but only on the integers $n_{i}, k, \ell \in \mathbb{Z}$.
} 
In this work, we have studied the Abelian SG flavor symmetry $D_{S}$ that arises from the constraints on closed strings to split and join while propagating on a six-dimensional orbifold, defined by a space group $S$. By demanding that $D_{S}$ be a (non-faithful) representation of $S$ and that the discrete charges of all closed strings be well-defined and conserved, we find that the SG flavor symmetry $D_{S}$ corresponds to the Abelianization of the space group $S$. We have discussed how this observation can be applied to space groups with different features, rendering the precise structure of $D_{S}$ and their charges for all closed strings.

In section 4 , we computed $D_{S}$ for all 138 space groups with Abelian point group that yield $\mathcal{N}=1$ effective field theories in four dimensions. It is known that the identified symmetries, displayed in table 1, play a key role as part of the flavor symmetries at low energies and are thus essential for phenomenology. As a cross check of the validity of these symmetries, we have also explicitly verified in thousands of $\mathrm{E}_{8} \times \mathrm{E}_{8}$ heterotic orbifold models that all identified $D_{S}$ lead to universal anomalies, which allows them to be canceled by a universal Green-Schwarz mechanism.

The SG flavor symmetries $D_{S}$ are respected in interactions of both massless and massive strings. Interestingly, there are cases where the charges of massless strings are restricted such that the symmetry of the massless sector is only a subgroup of $D_{S}$. An intriguing consequence is that the lightest massive string (which can be a winding mode) can only be produced and annihilated in pairs. Thus, the lightest massive string is stable and, hence, contributes to a dark sector of the effective model, see section 3.6.2. Whether this feature might be seen as an explanation of (some of) the dark matter of the universe and its evolution, or whether it rules out some of the orbifold geometries shall be studied elsewhere.

A natural extension of our work is the study of non-Abelian orbifolds, i.e. orbifolds whose point groups $P$ are non-Abelian [41-43]. One should explore how the constraints we imposed on the SG flavor symmetries $D_{S}$ and their charges apply to orbifolds with non-Abelian point groups, to answer whether this contribution to flavor symmetries is also Abelian in those scenarios.

\section{Acknowledgments}

S.R-S. was partly supported by DGAPA-PAPIIT grant IN100217 and CONACyT grants F252167 and 278017. P.V. is supported by the Deutsche Forschungsgemeinschaft (SFB1258). S.R-S. and P.V. would like to thank Hans Peter Nilles for discussions and the Bethe Center for Theoretical Physics in Bonn for hospitality and support.

Open Access. This article is distributed under the terms of the Creative Commons Attribution License (CC-BY 4.0), which permits any use, distribution and reproduction in any medium, provided the original author(s) and source are credited.

\section{References}

[1] L.J. Dixon, J.A. Harvey, C. Vafa and E. Witten, Strings on orbifolds, Nucl. Phys. B 261 (1985) 678 [INSPIRE]. 
[2] L.J. Dixon, J.A. Harvey, C. Vafa and E. Witten, Strings on orbifolds. 2, Nucl. Phys. B 274 (1986) 285 [INSPIRE].

[3] M. Blaszczyk, S. Groot Nibbelink, O. Loukas and S. Ramos-Sánchez, Non-supersymmetric heterotic model building, JHEP 10 (2014) 119 [arXiv:1407.6362] [INSPIRE].

[4] M. Blaszczyk, S. Groot Nibbelink, O. Loukas and F. Ruehle, Calabi-Yau compactifications of non-supersymmetric heterotic string theory, JHEP 10 (2015) 166 [arXiv:1507.06147] [INSPIRE].

[5] O. Lebedev et al., A mini-landscape of exact MSSM spectra in heterotic orbifolds, Phys. Lett. B 645 (2007) 88 [hep-th/0611095] [INSPIRE].

[6] O. Lebedev, H.P. Nilles, S. Ramos-Sánchez, M. Ratz and P.K.S. Vaudrevange, Heterotic mini-landscape. (II). Completing the search for MSSM vacua in a $Z_{6}$ orbifold, Phys. Lett. B 668 (2008) 331 [arXiv:0807.4384] [INSPIRE].

[7] D.K. Mayorga Peña, H.P. Nilles and P.-K. Oehlmann, A zip-code for quarks, leptons and Higgs bosons, JHEP 12 (2012) 024 [arXiv:1209.6041] [INSPIRE].

[8] S. Groot Nibbelink and O. Loukas, MSSM-like models on $Z_{8}$ toroidal orbifolds, JHEP 12 (2013) 044 [arXiv: 1308.5145] [INSPIRE].

[9] H.P. Nilles and P.K.S. Vaudrevange, Geography of fields in extra dimensions: string theory lessons for particle physics, Mod. Phys. Lett. A 30 (2015) 1530008 [arXiv:1403.1597] [INSPIRE].

[10] B. Carballo-Pérez, E. Peinado and S. Ramos-Sánchez, $\Delta$ (54) flavor phenomenology and strings, JHEP 12 (2016) 131 [arXiv:1607.06812] [INSPIRE].

[11] S. Ramos-Sánchez, On flavor symmetries of phenomenologically viable string compactifications, J. Phys. Conf. Ser. 912 (2017) 012011 [arXiv: 1708. 01595] [InSPIRE].

[12] Y. Olguín-Trejo, R. Pérez-Martínez and S. Ramos-Sánchez, Charting the flavor landscape of MSSM-like Abelian heterotic orbifolds, Phys. Rev. D 98 (2018) 106020 [arXiv:1808.06622] [INSPIRE].

[13] H.P. Nilles, S. Ramos-Sánchez, M. Ratz and P.K.S. Vaudrevange, A note on discrete $R$ symmetries in $Z_{6}$-II orbifolds with Wilson lines, Phys. Lett. B 726 (2013) 876 [arXiv: 1308.3435] [INSPIRE].

[14] N.G. Cabo Bizet, T. Kobayashi, D.K. Mayorga Peña, S.L. Parameswaran, M. Schmitz and I. Zavala, Discrete R-symmetries and anomaly universality in heterotic orbifolds, JHEP 02 (2014) 098 [arXiv: 1308.5669] [INSPIRE].

[15] H.P. Nilles, Stringy origin of discrete R-symmetries, PoS (CORFU2016) 017 (2017) [arXiv: 1705.01798] [INSPIRE].

[16] J. Lauer, J. Mas and H.P. Nilles, Duality and the role of nonperturbative effects on the world sheet, Phys. Lett. B 226 (1989) 251 [INSPIRE].

[17] J. Lauer, J. Mas and H.P. Nilles, Twisted sector representations of discrete background symmetries for two-dimensional orbifolds, Nucl. Phys. B 351 (1991) 353 [InSPIRE].

[18] L.E. Ibáñez and D. Lüst, Duality anomaly cancellation, minimal string unification and the effective low-energy Lagrangian of $4 D$ strings, Nucl. Phys. B 382 (1992) 305 [hep-th/9202046] [INSPIRE]. 
[19] D. Bailin, A. Love, W.A. Sabra and S. Thomas, Modular symmetries in $Z_{N}$ orbifold compactified string theories with Wilson lines, Mod. Phys. Lett. A 9 (1994) 1229 [hep-th/9312122] [INSPIRE].

[20] S. Hamidi and C. Vafa, Interactions on orbifolds, Nucl. Phys. B 279 (1987) 465 [INSPIRE].

[21] L.J. Dixon, D. Friedan, E.J. Martinec and S.H. Shenker, The conformal field theory of orbifolds, Nucl. Phys. B 282 (1987) 13 [INSPIRE].

[22] T. Kobayashi, H.P. Nilles, F. Plöger, S. Raby and M. Ratz, Stringy origin of non-Abelian discrete flavor symmetries, Nucl. Phys. B 768 (2007) 135 [hep-ph/0611020] [InSPIRE].

[23] H.P. Nilles, M. Ratz and P.K.S. Vaudrevange, Origin of family symmetries, Fortsch. Phys. 61 (2013) 493 [arXiv:1204.2206] [INSPIRE].

[24] H.P. Nilles, M. Ratz, A. Trautner and P.K.S. Vaudrevange, $C P$ violation from string theory, Phys. Lett. B 786 (2018) 283 [arXiv: 1808.07060] [InSPIRE].

[25] M. Fischer, M. Ratz, J. Torrado and P.K.S. Vaudrevange, Classification of symmetric toroidal orbifolds, JHEP 01 (2013) 084 [arXiv:1209.3906] [INSPIRE].

[26] M. Fischer, M. Ratz, J. Torrado and P.K.S. Vaudrevange, Classification of symmetric toroidal orbifolds webpage, http://users.ph.tum.de/ga57raj/Orbifolds/ClassificationOrbifolds/index.html.

[27] T. Araki, Anomaly of discrete symmetries and gauge coupling unification, Prog. Theor. Phys. 117 (2007) 1119 [hep-ph/0612306] [INSPIRE].

[28] W. Buchmüller, K. Hamaguchi, O. Lebedev and M. Ratz, Supersymmetric Standard Model from the heterotic string (II), Nucl. Phys. B 785 (2007) 149 [hep-th/0606187] [INSPIRE].

[29] J.G. Ratcliffe and S.T. Tschantz, Abelianization of space groups, Acta Cryst. A 65 (2008) 18.

[30] M. Blaszczyk, Heterotic particle models from various perspectives, Ph.D. thesis, University of Bonn, Bonn, Germany (2012).

[31] M. Blaszczyk and P.-K. Oehlmann, Tracing symmetries and their breakdown through phases of heterotic $(2,2)$ compactifications, JHEP 04 (2016) 068 [arXiv: 1512.03055] [INSPIRE].

[32] B. Petersen, M. Ratz and R. Schieren, Patterns of remnant discrete symmetries, JHEP 08 (2009) 111 [arXiv:0907.4049] [INSPIRE].

[33] R. Donagi and K. Wendland, On orbifolds and free fermion constructions, J. Geom. Phys. 59 (2009) 942 [arXiv: 0809.0330] [INSPIRE].

[34] S. Förste, T. Kobayashi, H. Ohki and K.-J. Takahashi, Non-factorisable $Z_{2} \times Z_{2}$ heterotic orbifold models and Yukawa couplings, JHEP 03 (2007) 011 [hep-th/0612044] [INSPIRE].

[35] M. Blaszczyk, S. Groot Nibbelink, M. Ratz, F. Ruehle, M. Trapletti and P.K.S. Vaudrevange, A $Z_{2} \times Z_{2}$ Standard Model, Phys. Lett. B 683 (2010) 340 [arXiv:0911.4905] [inSPIRE].

[36] H.P. Nilles, S. Ramos-Sánchez, P.K.S. Vaudrevange and A. Wingerter, The orbifolder: a tool to study the low energy effective theory of heterotic orbifolds, Comput. Phys. Commun. 183 (2012) 1363 [arXiv:1110.5229] [INSPIRE].

[37] K. Fujikawa, Path integral measure for gauge invariant fermion theories, Phys. Rev. Lett. 42 (1979) 1195 [INSPIRE].

[38] K. Fujikawa, Path integral for gauge theories with fermions, Phys. Rev. D 21 (1980) 2848 [Erratum ibid. D 22 (1980) 1499] [INSPIRE]. 
[39] T. Araki, T. Kobayashi, J. Kubo, S. Ramos-Sánchez, M. Ratz and P.K.S. Vaudrevange, (Non-)Abelian discrete anomalies, Nucl. Phys. B 805 (2008) 124 [arXiv:0805.0207] [INSPIRE].

[40] M.B. Green and J.H. Schwarz, Anomaly cancellation in supersymmetric D $=10$ gauge theory and superstring theory, Phys. Lett. B 149 (1984) 117 [INSPIRE].

[41] Z. Kakushadze, G. Shiu and S.-H. Henry Tye, Asymmetric non-Abelian orbifolds and model building, Phys. Rev. D 54 (1996) 7545 [hep-th/9607137] [INSPIRE].

[42] S.J.H. Konopka, Non Abelian orbifold compactifications of the heterotic string, JHEP 07 (2013) 023 [arXiv: 1210.5040] [INSPIRE].

[43] M. Fischer, S. Ramos-Sánchez and P.K.S. Vaudrevange, Heterotic non-Abelian orbifolds, JHEP 07 (2013) 080 [arXiv: 1304.7742] [INSPIRE]. 